\title{
GeV-scale inelastic dark matter with dark photon mediator via direct detection and cosmological and laboratory constraints
}

\author{
Hong-Jian He $\odot,{ }^{1,2,3, *}$ Yu-Chen Wang $\odot,{ }^{1,2, \dagger}$ and Jiaming Zheng $\oplus^{1, \$}$ \\ ${ }^{1}$ Tsung-Dao Lee Institute \& School of Physics and Astronomy, Key Laboratory for Particle Astrophysics \\ and Cosmology (MOE), Shanghai Key Laboratory for Particle Physics and Cosmology, Shanghai Jiao \\ Tong University, Shanghai 200240, China \\ ${ }^{2}$ Institute of Modern Physics \& Physics Department, Tsinghua University, Beijing 100084, China \\ ${ }^{3}$ Center for High Energy Physics, Peking University, Beijing 100871, China
}

(Received 17 January 2021; accepted 22 November 2021; published 29 December 2021)

\begin{abstract}
We propose a new candidate of GeV-scale inelastic dark matter (DM). Our construction has an anomalyfree $U(1)_{X}$ gauge group with a dark photon mediator, and it can realize either scalar or fermionic inelastic DM. It is highly predictive and testable. We study the scattering rate of light inelastic DM with electrons in the XENON1T experiment and with nuclei in the XENON1T, CRESST-III, CDEX-1B and DarkSide-50 experiments. We resolve the recent XENON1T anomaly via electron recoil detection. Combining the XENON1T constraints from both electron recoils and nuclear recoils (including the Migdal effect), we predict the inelastic DM mass $\lesssim 1.4 \mathrm{GeV}$. We further analyze the bounds by the DM relic abundance, the lifetime of heavier DM components, and laboratory constraints, from which we identify the viable parameter space for the future probe. This provides an important benchmark for the theories and experimental tests of $\mathrm{GeV}$-scale inelastic DM.
\end{abstract}

DOI: $10.1103 /$ PhysRevD.104.115033

\section{INTRODUCTION}

Searching for GeV-scale light dark matter (DM) particles is a very challenging task, because the conventional direct detection via DM-nucleon recoil becomes difficult for DM masses $\lesssim 5 \mathrm{GeV}$. Hence, measuring the DM-electron recoil spectrum has provided an important means for light DM direct detection. The XENON Collaboration [1] recently announced a $3.5 \sigma$ excess of events with low electron recoil energy in its Science Run I [2]. The XENON1T detector recorded 285 events for the recoil energy $E_{R}=(1-7) \mathrm{keV}$, among which the expected background events are $232 \pm 15$ [2]. This excess centers around $E_{R}=(2-3.5) \mathrm{keV}$. Lately, the PandaX-II Collaboration also reported an independent DM search by measuring the low-energy electron recoil spectrum with robust estimates of backgrounds [3]. It is consistent with the XENON1T measurement [2], although its sensitivity is not yet enough to either confirm or exclude the DM interpretation of the XENON1T anomaly. There have been possible explanations for this excess in the

\footnotetext{
*hjhe@sjtu.edu.cn; hjhe@tsinghua.edu.cn †wangyc21@sjtu.edu.cn

"zhengjm3@sjtu.edu.cn
}

Published by the American Physical Society under the terms of the Creative Commons Attribution 4.0 International license. Further distribution of this work must maintain attribution to the author(s) and the published article's title, journal citation, and DOI. Funded by SCOAP. literature, including an unexpected tritium background $[2,4]$ and various new physics models [5-8].

One attractive resolution of the XENON1T anomaly is the exothermic inelastic scattering $[7,8]$ between the DM and electrons. In this scenario, the DM consists of two components $\left(X, X^{\prime}\right)$ with a small mass splitting $\Delta m_{X} \equiv$ $m_{X^{\prime}}-m_{X}$, which is around the anomalous recoil energy region (2-3) keV of XENON1T. The heavier DM component $X^{\prime}$ is cosmologically stable, because its decay to the lighter DM component $X$ is highly suppressed by the small mass splitting $\Delta m_{X}$. Inside the xenon detector, $X^{\prime}$ scatters inelastically with the xenon electron and deexcites to $X$. ${ }^{1}$ The DM mass splitting manifests itself as a peak in the electron recoil energy spectrum. On the other hand, the recoil energy from the elastic DM-electron scattering is too small to be detectable in XENON1T unless the DM particles are very fast moving [5]. To generate the XENON1T anomaly, we can estimate the required normalized cross section of the DM-electron inelastic scattering, $\bar{\sigma}_{e} / m_{X} \approx 8.8 \times 10^{-44} \mathrm{~cm}^{2} / \mathrm{GeV}$, by considering the DM $\left(X, X^{\prime}\right)$ of equal amount and the DM-electron interaction of scalar or vector type [8]. Here we define $\bar{\sigma}_{e} \equiv \sigma_{X e}(|\mathbf{q}|=0)$ as the scattering cross section of $X^{\prime} e^{-} \rightarrow X e^{-}$in the zeromomentum-exchange limit $|\mathbf{q}|=0$.

\footnotetext{
${ }^{1}$ This differs in an essential way from the well-studied endothermic inelastic DM scattering in the literature [9], which cannot explain the XENON1T anomaly and is irrelevant to the our current work.
} 
In the literature, the inelastic scatterings mostly arise from exchanging a light dark photon with couplings to leptons by assuming a tiny kinetic mixing between the dark photon and the standard-model (SM) photon [7]. These models require a large hierarchy between the dark-photonlepton coupling and the dark-photon-DM coupling. So, the dark photons could be hidden from various collider searches by tuning the kinetic mixing of the dark photon with the photon down to $O\left(10^{-3}-10^{-6}\right)$. The origin of such tiny kinetic mixing remains obscure [10], so we will not pursue this for the present study.

In this work, we propose a new candidate of $\mathrm{GeV}$-scale inelastic DM. We realize this in an anomaly-free and renormalizable model, in which the DM and the right-handed first-family fermions join the interactions of a dark $U(1)_{X}$ gauge group. We achieve the desired $O(\mathrm{keV})$ mass splitting of the inelastic DM by a scalar seesaw mechanism without fine-tuning. We show that this minimal model is viable for the inelastic DM of mass $\lesssim 1.4 \mathrm{GeV}$, which can provide the intriguing anomaly in the XENON1T electron recoil spectrum [2] and be consistent with the DMnucleon recoil detections (of low threshold) by XENON1T [11], CRESST-III [12], CDEX-1B [13], and DarkSide-50 [14] experiments. For the dark photon mediator with mass $<2 m_{X}$, this model can provide the observed DM relic abundance and ensure that the heavier DM component is cosmologically stable. We further derive nontrivial bounds from the existing laboratory measurements, including the electroweak precision tests and collider searches. We also discuss the possible future experimental probes.

This paper is organized as follows: We construct our model in Sec. II. Then, in Sec. III, we analyze the DMelectron and DM-nucleon recoil signals in various directdetection experiments. In Sec. IV, we study the cosmological constraints on our model, including the $X^{\prime}$ lifetime and the DM relic abundance. In Sec. V, we study other laboratory constraints, including the electroweak precision tests and the collider searches. Finally, we conclude in Sec. VI. In Appendix A, we propose an improved treatment of the Migdal effect bound on the inelastic DM. Appendix B presents our analysis on the Higgs sector of this model.

\section{INELASTIC DM WITH DARK PHOTON MEDIATOR}

To realize the DM-electron interaction, we construct a minimal extension of the SM by a dark $U(1)_{X}$ gauge group under which both the DM and the right-handed first-family fermions are charged. We also include three right-handed Majorana neutrinos $\nu_{R j}(j=1,2,3)$. We denote the $U(1)_{X}$ gauge boson (dark photon) by $A_{\mu}^{\prime}$. The Higgs sector consists of two Higgs doublets plus three singlet scalars $S, S^{\prime}$, and $\phi$, charged under $U(1)_{X}$. The electroweak symmetry breaking is realized by two Higgs doublets $H_{1}$ and $H_{2}$ with vacuum expectation values (VEVs) $\left\langle H_{j}\right\rangle=\left(0, v_{j}\right)^{T}$ and their
TABLE I. Particle content and group assignments of our model. Here $Q_{L_{1}}\left(L_{1}\right)$ denotes the left-handed weak doublet of quarks (leptons) in the first family of the SM, while the second and third families of the SM fermions are $U(1)_{X}$ singlets and $\mathbb{Z}_{2}$-even. The second column from the right defines the scalar inelastic DM $\hat{X}$, and the last column defines the fermionic inelastic DM $\hat{\chi}$ as another setup.

\begin{tabular}{lccccccccccccc}
\hline \hline Group & $Q_{L 1}$ & $u_{R}$ & $d_{R}$ & $L_{1}$ & $e_{R}$ & $\nu_{R}$ & $H_{1}$ & $H_{2}$ & $S$ & $S^{\prime}$ & $\phi$ & $\hat{X}$ & $\hat{\chi}$ \\
\hline$S U(2)_{L}$ & $\mathbf{2}$ & $\mathbf{1}$ & $\mathbf{1}$ & $\mathbf{2}$ & $\mathbf{1}$ & $\mathbf{1}$ & $\mathbf{2}$ & $\mathbf{2}$ & $\mathbf{1}$ & $\mathbf{1}$ & $\mathbf{1}$ & $\mathbf{1}$ & $\mathbf{1}$ \\
$U(1)_{Y}$ & $\frac{1}{6}$ & $\frac{2}{3}$ & $-\frac{1}{3}$ & $-\frac{1}{2}$ & -1 & 0 & $\frac{1}{2}$ & $\frac{1}{2}$ & 0 & 0 & 0 & 0 & 0 \\
$U(1)_{X}$ & 0 & $\frac{1}{2}$ & $-\frac{1}{2}$ & 0 & $-\frac{1}{2}$ & $\frac{1}{2}$ & $\frac{1}{2}$ & 0 & -1 & $\frac{1}{2}$ & -3 & 3 & $\frac{3}{2}$ \\
$\mathbb{Z}_{2}$ & + & + & + & + & + & + & + & + & + & + & + & - & - \\
\hline \hline
\end{tabular}

combined VEV $v_{h}=\sqrt{v_{1}^{2}+v_{2}^{2}} \simeq 174 \mathrm{GeV}$. We will set $v_{1}^{2} \ll v_{2}^{2}$, so the observed Higgs boson $(125 \mathrm{GeV})$ is mostly made of the $C P$-even neutral component of $\mathrm{H}_{2}$. The dark $U(1)_{X}$ gauge group is mainly broken by the VEVs of the singlet scalars $S$ and $S^{\prime}$, whose VEVs $\langle S\rangle=v_{S}$ and $\left\langle S^{\prime}\right\rangle=$ $v_{S}^{\prime}$ are of $O(100 \mathrm{GeV})$. Our model sets the first-family fermions charged under $U(1)_{X}$, and the second- and third-family fermions as $U(1)_{X}$ singlets. In the following subsections, we will study the case of scalar DM $\hat{X}=$ $\left(X+i X^{\prime}\right) / \sqrt{2}$ and the case of fermionic DM $\hat{\chi}=$ $\left(\chi_{1}, \chi_{2}^{\dagger}\right)^{T}$, respectively. In Table I, we present the particle content and charge assignments of our model for the dark sector, the Higgs sector, and the first-family fermions.

We note that in the lepton sector, only the right-handed $\left(e_{R}, \nu_{R 1}\right)$ are charged under $U(1)_{X}$ to maintain the stability of the heavier DM component. The $U(1)_{X}$ charge assignments of the first-family SM fermions (including the right-handed neutrino) are then uniquely determined by the anomaly cancellation.

\section{A. Inelastic scalar DM with dark $U(1)_{X}$}

The DM particles $\left(X, X^{\prime}\right)$ form a complex scalar $\hat{X}=$ $\left(X+i X^{\prime}\right) / \sqrt{2}$ with $U(1)_{X}$ charge $q_{\hat{X}}$. As we will show, the spontaneous breaking of $U(1)_{X}$ will generate the desired mass splitting $\Delta m_{X}$ between $X$ and $X^{\prime}$. Our model sets the left-handed fermion doublets as $U(1)_{X}$ singlets. This forbids the decay channel $X^{\prime} \rightarrow X \bar{\nu} \nu$, and thus ensures that the current DM relic abundance consists of about equal amounts of $\left(X, X^{\prime}\right)$ so far. The anomaly cancellation conditions then uniquely determine the $U(1)_{X}$ charges of the first-family fermions up to an overall normalization factor. The flavor nonuniversality of $U(1)_{X}$ ensures $H_{2}$ as a $U(1)_{X}$ singlet, so the $A^{\prime}-Z$ mixing is suppressed by $v_{1}^{2} / v_{h}^{2} \ll 1$ and thus experimentally viable, as will be shown in Sec. IV.

We write down the relevant Lagrangian terms of the DM sector as follows:

$$
\begin{aligned}
\Delta \mathcal{L}_{\mathrm{DM}} \supset & \left|D^{\mu} \hat{X}\right|^{2}-m_{\hat{X}}^{2}|\hat{X}|^{2}-\lambda_{X}|\hat{X}|^{4} \\
& -\left(\lambda_{X \phi} \hat{X}^{2} \phi^{2}+\text { H.c. }\right)-\sum_{i} \lambda_{X \psi_{i}}|\hat{X}|^{2}\left|\psi_{i}\right|^{2},
\end{aligned}
$$


where the scalar fields $\psi_{i}=H_{1}, H_{2}, S, S^{\prime}, \phi$. According to Table I, $H_{1}$ only couples to the first-family fermions, while $\mathrm{H}_{2}$ interacts only with the second and third families of fermions. Thus, we can write the Lagrangian including the relevant Yukawa terms with $\nu_{R j}$ and the relevant potential terms with scalar singlets:

$$
\begin{aligned}
\Delta \mathcal{L} \supset \bar{e}_{R} i \not e_{R}-\sum_{i=1}^{3}\left(y_{i 1}^{\nu} \bar{L}_{i} \tilde{H}_{1} \nu_{R 1}+\sum_{j=2}^{3} y_{i j}^{\nu} \bar{L}_{i} \tilde{H}_{2} \nu_{R j}+\text { H.c. }\right) \\
\quad-\frac{1}{2}\left(y_{S} \nu_{R 1}^{T} S \nu_{R 1}+\sum_{i, j=2}^{3} M_{R i j} \nu_{R i}^{T} \nu_{R j}+\text { H.c. }\right) \\
\quad+M_{S}^{2}|S|^{2}+\left.M_{S^{\prime}}^{2} S^{\prime}\right|^{2}+\left(M_{12}^{\prime} H_{1}^{\dagger} H_{2} S^{\prime}+\text { H.c. }\right) \\
\quad-M_{\phi}^{2}|\phi|^{2}+\left(\lambda_{S \phi} S^{3} \phi^{*}+\text { H.c. }\right) .
\end{aligned}
$$

We note that the cubic term $M_{12}^{\prime} H_{1}^{\dagger} H_{2} S^{\prime}$ can ensure the pseudoscalars to be massive. In Eq. (2), the squared masses $\left(M_{S}^{2}, M_{S^{\prime}}^{2}, M_{\phi}^{2}\right)$ are all positive, so $S$ and $S^{\prime}$ acquire VEVs from their potentials directly, whereas $\phi$ can only obtain a small VEV induced from $\langle S\rangle$ and $\left\langle S^{\prime}\right\rangle$. In our model, the scalar potential holds $C P$ symmetry, under which all the scalar couplings and VEVs are real. Equation (2) shows that the singlet $S$ and the right-handed neutrino $\nu_{R 1}$ form a Yukawa interaction which generates a weak scale Majorana mass $M_{R 1}=y_{S 1} v_{S}$ for $\nu_{R 1}$. The second- and third-family right-handed neutrinos are $U(1)_{X}$ singlets, so they form Majorana mass terms directly. Thus, the light neutrino masses are generated by the type-I seesaw mechanism.

From the Lagrangian (1), we see that the DM mass is determined by the DM quadratic mass term and the DM couplings to $\left|H_{i}\right|^{2},|S|^{2}$, and $\left|S^{\prime}\right|^{2}$. The mass splitting between the two DM components is determined by the unique quartic interaction $\hat{X}^{2} \phi^{2}$, where the singlet $\operatorname{VEV}\langle\phi\rangle$ is naturally small as generated by a scalar seesaw ${ }^{2}$ from the potential terms in the last line of Eq. (2). This is because $\phi$ is much heavier than all the other scalars and leads to $v_{\phi} \equiv\langle\phi\rangle \simeq \lambda_{S \phi} v_{S}^{3} / M_{\phi}^{2}$. Thus, we derive the $\left(X, X^{\prime}\right)$ mass splitting:

$$
\frac{m_{X^{\prime}}-m_{X}}{m_{X}} \simeq \frac{\lambda_{X \phi} v_{\phi}^{2}}{m_{X}^{2}}=\frac{2 \lambda_{X \phi} \lambda_{S \phi}^{2} v_{S}^{6}}{m_{X}^{2} M_{\phi}^{4}} .
$$

Hence, to realize the desired $O(\mathrm{keV})$ mass splitting for explaining the XENON1T anomaly, we can choose the sample inputs without fine-tuning: $\lambda_{X \phi}, \lambda_{S \phi}=O(0.01)$, $v_{S}=O(100) \mathrm{GeV}, m_{X}=O(\mathrm{GeV})$, and $M_{\phi}=O(\mathrm{TeV})$. This gives $v_{\phi}=O(10) \mathrm{MeV}$ and $\Delta m_{X}=O(\mathrm{keV})$.

\footnotetext{
${ }^{2}$ The realization of this scalar seesaw mechanism requires the presence of a $U(1)$ symmetry. It is truly attractive and economical to identify it as a gauge symmetry $U(1)_{X}$ whose gauge boson serves as the portal (mediator) between the dark and visible sectors.
}

Since the Higgs doublet $H_{1}$ carries charges of both $U(1)_{Y}$ and $U(1)_{X}$, its VEV induces mass mixing between their gauge bosons. Denoting the neutral gauge bosons of $S U(2)_{L}, U(1)_{Y}$, and $U(1)_{X}$ as $\left(W_{\mu}^{3}, B_{\mu}, \mathcal{X}_{\mu}\right)$, we derive their mass eigenstates $\left(Z_{\mu}, A_{\mu}, A_{\mu}^{\prime}\right)$ to the leading order of the gauge coupling $g_{X} \ll 1$ and VEV ratio $v_{1}^{2} / v_{h}^{2} \ll 1$, with the mass eigenvalues

$$
\begin{aligned}
& m_{A}^{2}=0, \\
& m_{A^{\prime}}^{2} \simeq 2 g_{X}^{2}\left(v_{S}^{2}+\frac{1}{4} v_{S^{\prime}}^{2}\right), \\
& M_{Z}^{2} \simeq \frac{1}{2}\left(g^{2}+g^{\prime 2}+g_{X}^{2} \frac{v_{1}^{4}}{v_{h}^{4}}\right) v_{h}^{2},
\end{aligned}
$$

and their leading-order mixing matrix,

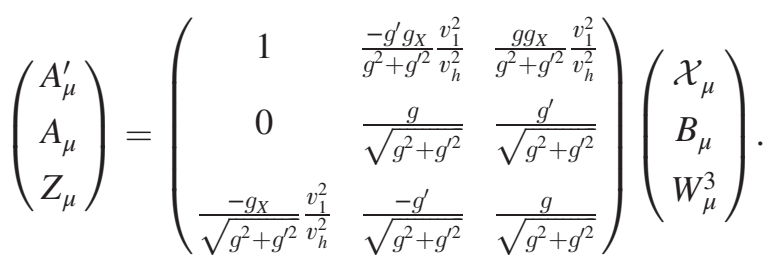

\section{B. Inelastic fermionic DM with dark $U(1)_{X}$}

The mechanism of realizing $O(\mathrm{keV})$ mass splitting for the scalar inelastic DM in Sec. II A can be extended to the case of inelastic fermionic DM. In this subsection, we present a construction of inelastic fermionic DM. In this model, the charge assignments (Table I) and the scalar potential [Eq. (2)] remain the same as before, except that the fermionic $\mathrm{DM} \hat{\chi}$ has a different $U(1)_{X}$ charge, as shown in the last column of Table I. The fermionic DM contains two Weyl spinors $\chi_{1}$ and $\chi_{2}$, with opposite $U(1)_{X}$ charges $q_{\chi_{1}}=-q_{\chi_{2}}=\frac{3}{2}$. They can form a Dirac spinor $\hat{\chi}=\left(\chi_{1}, \chi_{2}^{\dagger}\right)^{T}$, which is vectorlike under $U(1)_{X}$. Thus, the dark sector contains the following gauge-invariant Lagrangian terms:

$$
\begin{gathered}
\Delta \mathcal{L} \supset \chi_{1}^{\dagger} i \bar{\sigma}^{\mu} D_{\mu} \chi_{1}+\chi_{2}^{\dagger} i \bar{\sigma}^{\mu} D_{\mu} \chi_{2}-\left(m_{\hat{\chi}} \chi_{1} \chi_{2}+\text { H.c. }\right) \\
+\left(y_{\phi \chi_{1}} \chi_{1} \chi_{1} \phi+y_{\phi \chi_{2}} \chi_{2} \chi_{2} \phi^{*}+\text { H.c. }\right)
\end{gathered}
$$

where the parameters $\left(m_{\hat{\chi}}, y_{\phi \chi_{1}}, y_{\phi \chi_{2}}\right)$ are positive after proper phase rotations of $\left(\phi, \chi_{1}, \chi_{2}\right)$. As shown in Sec. II A, the scalar field $\phi$ acquires a small VEV $v_{\phi} \simeq \lambda_{S \phi} v_{S}^{3} / M_{\phi}^{2}$ due to the scalar seesaw from Eq. (2). The VEV $v_{\phi}$ will induce additional Majorana masses for $\left(\chi_{1}, \chi_{2}\right)$ through the Yukawa interactions in Eq. (6). Thus, we have the following DM mass terms:

$$
\mathcal{L}_{\chi_{1} \chi_{2}} \supset-m_{\hat{\chi}} \chi_{1} \chi_{2}+\delta m_{1} \chi_{1} \chi_{1}+\delta m_{2} \chi_{2} \chi_{2}+\text { H.c., }
$$

where $\left(\delta m_{1}, \delta m_{2}\right)=\left(y_{\phi \chi_{1}} v_{\phi}, y_{\phi \chi_{2}} v_{\phi}\right)$. To rotate $\left(\chi_{1}, \chi_{2}\right)$ into the mass eigenstates $\left(\chi, \chi^{\prime}\right)$, we make the following decomposition: 


$$
\chi_{1} \simeq \frac{1}{\sqrt{2}}\left(\chi-i \chi^{\prime}\right), \quad \chi_{2} \simeq \frac{1}{\sqrt{2}}\left(\chi+i \chi^{\prime}\right) .
$$

In the limit $v_{\phi} \ll m_{\hat{\chi}}$, we derive the following Majorana masses for the DM mass eigenstates $\left(\chi, \chi^{\prime}\right)$ :

$$
\begin{aligned}
& m_{\chi} \simeq m_{\hat{\chi}}-\left(\delta m_{1}+\delta m_{2}\right), \\
& m_{\chi^{\prime}} \simeq m_{\hat{\chi}}+\left(\delta m_{1}+\delta m_{2}\right),
\end{aligned}
$$

which have a mass splitting

$$
\Delta m_{\chi} \simeq 2\left(\delta m_{1}+\delta m_{2}\right) .
$$

To realize the required mass splitting of $O(\mathrm{keV})$ for explaining the XENON1T anomaly, we choose the natural sample inputs, $\lambda_{S \phi}, y_{\phi \chi_{1}}, y_{\phi \chi_{2}}=O(0.01), v_{S}=O(20) \mathrm{GeV}$, and $M_{\phi}=O(\mathrm{TeV})$. With these, we deduce a small VEV of $v_{\phi}=O(0.1) \mathrm{MeV}$, and thus the desired mass splitting $\Delta m_{\chi}=O(\mathrm{keV})$. From Eq. (6), we deduce the $U(1)_{X}$ gauge interactions for the DM fields $\left(\chi, \chi^{\prime}\right)$ :

$$
\mathcal{L}_{\text {int }} \supset i q_{\hat{\chi}} g_{X}\left(\chi^{\dagger} \bar{\sigma}_{\mu} \chi^{\prime}-\chi^{\prime \dagger} \bar{\sigma}_{\mu} \chi\right) \mathcal{X}^{\mu} .
$$

We note that the diagonal vertices $\chi-\chi-\mathcal{X}^{\mu}$ and $\chi^{\prime}-\chi^{\prime}-\mathcal{X}^{\mu}$ vanish, whereas the above nondiagonal vertices can induce the desired inelastic scattering for explaining the XENON1T anomaly of DM-electron recoils.

Most discussions in the rest of this paper can apply to both the scalar and fermionic DM. For the simplicity of notation, unless specified otherwise, we will use the same symbols $\left(\Delta m_{X}, m_{X}\right)$ and $\left(\hat{X}, X, X^{\prime}\right)$ for both scalar and fermionic DM. For the fermionic DM, these notations refer to $\left(\Delta m_{\chi}, m_{\hat{\chi}}\right)$ and $\left(\hat{\chi}, \chi, \chi^{\prime}\right)$.

\section{ANALYZING CONSTRAINTS OF DM DIRECT DETECTIONS}

For the present study, we derive the DM-electron inelastic scattering cross section with zero momentum transfer, which holds for both scalar DM and fermionic DM:

$$
\bar{\sigma}_{e} \equiv \sigma_{X e}(|\mathbf{q}|=0)=\frac{q_{e}^{2} q_{\mathrm{DM}}^{2} g_{X}^{4}}{4 \pi} \frac{m_{e}^{2}}{m_{A^{\prime}}^{4}},
$$

where $e_{R}$ carries $U(1)_{X}$ charge $q_{e}=-\frac{1}{2}$. The $U(1)_{X}$ charge of the scalar DM is $q_{\mathrm{DM}}=q_{\hat{X}}=3$, and the fermionic DM has $q_{\mathrm{DM}}=q_{\hat{\chi}}=\frac{3}{2}$. In Ref. [8], we proposed an effective field theory (EFT) approach to perform a model-independent fit of the inelastic DM for the XENON1T electron recoil spectrum [2]. Our fit shows that the XENON1T anomaly [2] can be fully explained by the two-component inelastic DM with mass splitting $\Delta m_{X} \equiv m_{X^{\prime}}-m_{X}=2.8_{-0.3}^{+0.2} \mathrm{keV}$ (68\% C.L.) and $2.1 \mathrm{keV}<\Delta m_{X}<3.3 \mathrm{keV}$ (95\% C.L.). We obtain the ratio of the inelastic cross section over the DM mass, $\bar{\sigma}_{e} / m_{X}=(8.8 \pm 4.0) \times 10^{-44} \mathrm{~cm}^{2} / \mathrm{GeV}$, under the condition that the DM density contains an equal amount of $X$ and $X^{\prime}$ particles. Thus, by fitting the XENON1T data [2], we derive the following bound on our model:

$$
\frac{m_{A^{\prime}}}{g_{X}}=1.2_{-0.1}^{+0.2} \times 10^{2} \mathrm{GeV} \times\left[\left|\frac{q_{e} q_{\mathrm{DM}}}{3 / 2}\right|^{\frac{1}{2}}\left(\frac{1 \mathrm{GeV}}{m_{X}}\right)^{\frac{1}{4}}\right] .
$$

In Fig. 1(a), we present the electron recoil energy spectrum as predicted by the inelastic DM, in comparison with that of the XENON1T measurement [2]. The recoil spectra plotted in the (green, red, blue) dashed curves correspond to the inelastic DM contributions with mass splitting $\Delta m_{X}=(2.5,2.8,3.0) \mathrm{keV}$, whereas the (green, red, blue) solid curves further include the background contribution as given by model $B_{0}$ of XENON1T [2] (depicted by the black solid curve). The data points with error bars show the XENON1T measurement [2]. We have input a sample cross-section/mass ratio $\bar{\sigma}_{e} / m_{X}=8.8 \times$ $10^{-44} \mathrm{~cm}^{2} / \mathrm{GeV}$, which is the best fit with XENON1T data [2], as mentioned above Eq. (13). We note that the shape of our fitted recoil peak is mainly determined by the data points below $5 \mathrm{keV}$, and the fit with the spectrum above $5 \mathrm{keV}$ has little effect on the shape of this peak. The background model $B_{0}$ is fitted to the recoil energy data over a very wide range of (1-210) $\mathrm{keV}$ [2], hence its normalization has negligible fluctuation.

In Fig. 1(b), we show $m_{A^{\prime}} /\left(g_{X} \sqrt{q_{\mathrm{DM}}}\right)$ as a function of the DM mass $m_{X}$. By fitting the recent XENON1T data [2], we present the allowed parameter space by the pink area at 95\% C.L., and we plot the central values (best fit) by the red solid curve. The PandaX-II Collaboration has accumulated 100.7 ton-day data from measuring the electron recoil spectrum [3], which is consistent with the background fluctuations. In each bin of the PandaX-II data, we find the expected DM signal rate to be less than 10 events $/ \mathrm{keV}$, which is smaller than the error bar. Hence, the best fit of our model is consistent with PandaX-II, although its current bound is too weak to be shown in Fig. 1.

The DM particles in our model can also scatter with nuclei by exchanging the mediator $A_{\mu}^{\prime}$, since the quarks $\left(u_{R}, d_{R}\right)$ carry $U(1)_{X}$ charges as in Table I. The DMnucleon scattering cross section is dominated by the vector coupling of $A_{\mu}^{\prime}$ with quarks, since the contribution of the axial-vector coupling is suppressed by the velocity of the recoiled nucleus. Thus, the spin-independent contribution dominates the DM-nucleon scattering. The $U(1)_{X}$ charge of a nucleon is the sum of its valence quarks at low energy-i.e., $q_{p}=\frac{1}{2}$ for a proton and $q_{n}=-\frac{1}{2}$ for a neutron. We thus compute the DM-nucleon scattering cross section as follows $[15,16]$ :

$$
\sigma_{X N}=\frac{m_{N}^{2} m_{X}^{2} q_{N}^{2} q_{\mathrm{DM}}^{2} g_{X}^{4}}{4 \pi\left(m_{X}+m_{N}\right)^{2} m_{A^{\prime}}^{4}},
$$



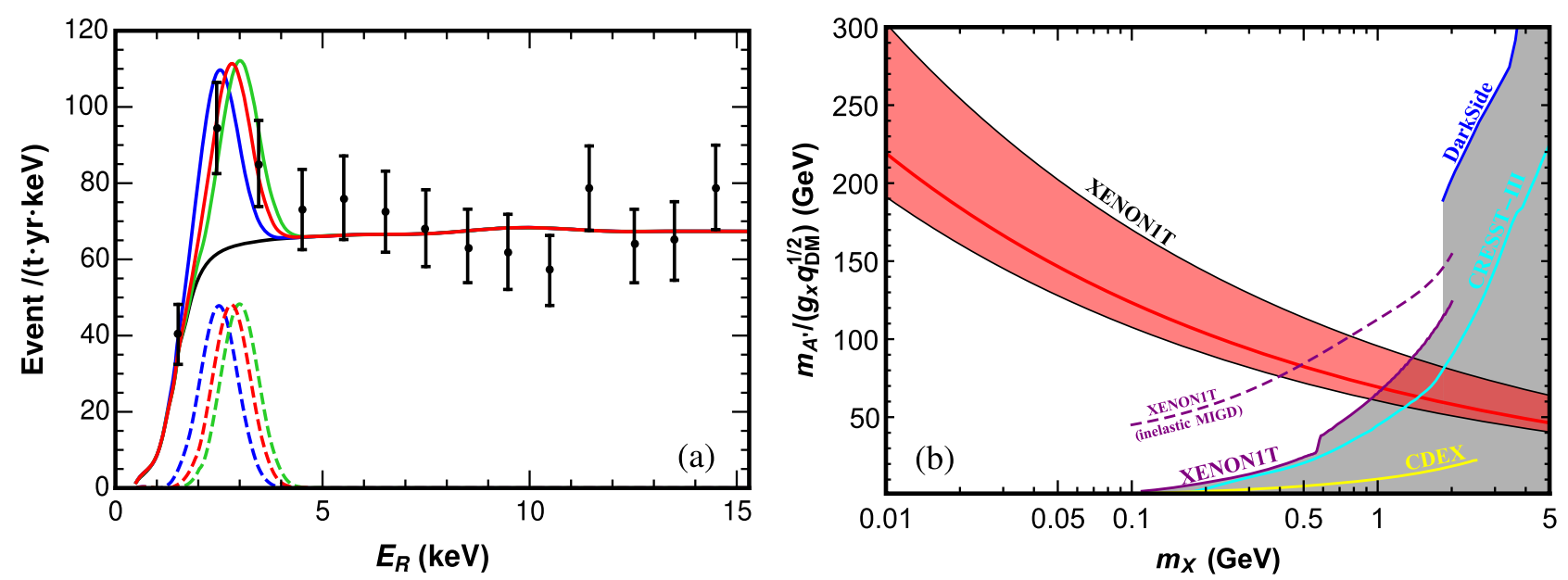

FIG. 1. (a) Electron recoil energy spectrum from electron-DM scattering as predicted by the inelastic DM and measured by XENON1T [2]. The (green, red, blue) dashed curves present the inelastic DM contributions with the DM mass splitting $\Delta m_{X}=(2.5,2.8,3.0) \mathrm{keV}$, while the (green, red, blue) solid curves further include the XENON1T background contribution (shown as the black solid curve). The data points with error bars show the XENON1T measurement [2]. (b) Constraints on our model by the direct DM detections of XENON1T, CRESST-III, CDEX-1B, and DarkSide. The pink area shows the allowed region (95\% C.L.), and the red solid curve gives the central value from fitting the XENON1T electron recoil data [2]. The gray regions are excluded at 90\% C.L. by the nucleus recoil detections (of low threshold), where the (purple, light blue, green, dark blue) curves present the existing exclusion limits from the XENON1T [11], CRESST-III [12], CDEX-1B [13], and DarkSide-50 [14] experiments via nucleon recoils, respectively. The purple dashed curve presents the estimate of an improved constraint of the Migdal effect on the inelastic DM inferred from the XENON1T result [11].

where $m_{N} \simeq 940 \mathrm{MeV}$ is the mass of a nucleon, and $q_{N}=\frac{1}{2}$ $\left(-\frac{1}{2}\right)$ for protons (neutrons). The above formula of the DMnucleon scattering cross section remains the same for both scalar and fermionic DM, except that the $U(1)_{X}$ charge $q_{\mathrm{DM}}$ of the DM can differ in the two cases. This is consistent with the fact that the dominant contribution to the scattering is spin independent and does not rely on the mass splitting $\Delta m_{X}$. If such scattering could flip the spin of the fermionic DM, it must proceed by changing either the orbital angular momentum of the system or the spin state of the nucleus. The amplitude of the former is suppressed by the low DM velocity, while the latter depends on the nuclear spin and thus is much smaller than the dominant spin-independent contribution. The leading amplitude is thus diagonal in the space of DM spin states, and it is proportional to $J_{X}^{\mu}=\left\langle X\left(k_{2}\right)\left|\hat{J}_{X}^{\mu}\right| X^{\prime}\left(k_{1}\right)\right\rangle \propto q_{\mathrm{DM}}\left(k_{1}^{\mu}+k_{2}^{\mu}\right)+O\left(\Delta m_{X}\right)$. This form is fixed by the $U(1)_{X}$ current conservation $q_{\mu} J_{X}^{\mu}=0$ in the limit $\Delta m_{X} \rightsquigarrow 0$, where the momentum transfer $q^{\mu}=k_{1}^{\mu}-k_{2}^{\mu}$. This form does not depend on the spin of DM; hence, the leading term of the DM-nucleus scattering cross section remains the same for both scalar and fermionic DM.

We also note that the $U(1)_{X}$ charges of protons and neutrons have opposite signs in our model. This gives the total $U(1)_{X}$ charge of a nucleus $Q=\frac{1}{2}(A-2 Z)$, where $A$ and $Z$ denote the nucleon number and proton number, respectively. The form factor is nearly constant, $f\left(q r_{\mathrm{n}}\right) \simeq 1$, because for the light DM $m_{X}<5 \mathrm{GeV}$, the momentum transfer $q \lesssim O(10 \mathrm{MeV})$ is small. Thus, to compare our model with the nuclear recoil measurements which usually assume isospin symmetry between protons and neutrons for spin-independent interactions, we can properly rescale their original bounds on the DM-nucleon cross section by a factor of $A^{2} /(A-2 Z)^{2}$, where a weighted average over isotope abundance is understood.

For dual-phase detectors such as XENON1T [17] and DarkSide-50 [18], the nuclear recoil causes primary scintillation signals that are measured with energy thresholds at several $\mathrm{keV}$, which are sensitive to DM particles with masses $\gtrsim 5 \mathrm{GeV}$. Due to the Migdal effect [19], the recoiled nuclei produce ionization and/or excitation of their atomic electrons with finite probability. The secondary radiation signals created by these electrons can be detected with much lower energy thresholds $(\lesssim 1 \mathrm{keV})$, and thus largely enhance the sensitivity to sub-GeV DM. These effects have been included in the direct-detection experimental papers such as Ref. [11]. The differential rate of electron recoil (ER) in the Migdal process accompanying a nucleus recoil (NR) is given by $[11,19]$

$$
\frac{\mathrm{d} R}{\mathrm{~d} E_{\mathrm{ER}}} \simeq \int \mathrm{d} E_{\mathrm{NR}} \mathrm{d} v\left[\frac{\mathrm{d}^{2} R_{\mathrm{N}}}{\mathrm{d} E_{\mathrm{NR}} \mathrm{d} v} \sum_{n, \ell} \frac{\mathrm{d} P_{q_{e}}^{c}}{2 \pi \mathrm{d} E_{\mathrm{ER}}}\right]
$$

where $P_{q_{e}}^{c}$ encodes the probability for the ionization of atomic electron with quantum numbers $(n, \ell)$ suppressed in the expression. The differential rate of the nuclear recoil, $\frac{\mathrm{d}^{2} R_{\mathrm{N}}}{\mathrm{d} E_{\mathrm{NR}} \mathrm{d} v}$, depends only on the short-scale particle physics 
model following the standard calculation of DM-nuclear scattering. Because of the clear separation of the nuclear and atomic physics effects as in Eq. (15), the XENON1T Collaboration already took into account the atomic ionization factor $P_{q_{e}}^{c}$ and transferred its limit on the electron recoil event rate to the limit on the DM-nucleon elastic cross section. For exothermic inelastic scattering, the energy release from the DM makes it easier to excite Migdal electrons than elastic scattering, as shown in Ref. [20]. Thus, we can view the XENON1T result [11] on DMnucleus elastic scattering as a conservative constraint on Eq. (14). So far in the literature, the constraints of including the Migdal effect are derived only for elastic DM [11] and for inelastic DM with a few special inputs of $\delta$ [20]. In Appendix A, we make an estimate of the improved constraint of the Migdal effect on the inelastic DM by using the XENON1T result [11].

In the following, we summarize the best constraints on DM-nucleon scattering from current direct-detection experiments. The strongest bound is set by the recent XENON1T detection [11], which probes the light DM with masses down to about $85 \mathrm{MeV}$ by measuring electronic recoils induced by the Migdal effect and bremsstrahlung. It can detect both scintillation and ionization signals, as well as ionization signals only, which allows for a lower detection threshold. ${ }^{3}$ The recent DarkSide-50 measurement [14] used a target of low-radioactivity argon and analyzed the ionization signals, which probes the light DM mass down to the (1.8-6) GeV range. The CDEX-1B experiment [13] uses $P$-type point contact germanium (PPCGe) detectors to detect both the nuclear recoil energy and electron ionization energy. It can probe the light DM mass down to $50 \mathrm{MeV}$. Utilizing solid-state detectors with low energy thresholds is another significant means for light DM detection. The CRESST-III detection [12] operates scintillating $\mathrm{CaWO}_{4}$ crystals as cryogenic calorimeters and can achieve a low nuclear recoil threshold energy of $30.1 \mathrm{eV}$. It is sensitive to light DM of mass below $\sim 2 \mathrm{GeV}$.

In Fig. 1(b), we present the bounds (90\% C.L.) from XENON1T [11], CRESST-III [12], CDEX-1B [13], and DarkSide-50 [14] on the parameter space of our model, shown by the gray region, over the mass range $m_{X}=(0.01-5) \mathrm{GeV}$. Here the purple, light blue, yellow, and dark blue curves give the $90 \%$ exclusion limits set by the XENON1T, CRESST-III, CDEX-1B, and DarkSide-50 experiments, respectively. We find that the XENON1T measurement (including the Migdal effect) imposes the strongest limit on our parameter space among the existing

\footnotetext{
${ }^{3}$ We thank our experimental colleagues Qing Lin, Kaixuan Ni, and Jingqiang Ye in the XENON1T Collaboration [11] for discussing their analysis of including the Migdal effect and confirming our application of their bound from Fig. 5 of Ref. [11]. We also thank Qian Yue of the CDEX-1B Collaboration for confirming our application of their experimental bound [13] including the Migdal effect.
}

bounds. ${ }^{4}$ This constrains the inelastic DM mass within the range $m_{X} \lesssim 1.4 \mathrm{GeV}$. The purple dashed curve shows our estimated constraint of the Migdal effect on the (exothermic) inelastic DM inferred from the original XENON1T result [11] on elastic DM based on the scaling relation of Eq. (A6). We see that this improved analysis could enhance the constraint to around $m_{X} \lesssim 0.8 \mathrm{GeV}$. We note that in general, using the Migdal effect can place strong constraints on inelastic DM, and our new method can be applied to a broad class of inelastic DM models. We will perform a systematic analysis of the Migdal effect on various inelastic DM models in future work.

\section{ANALYZING COSMOLOGICAL CONSTRAINTS}

In this section, we analyze the relevant cosmological constraints on the present inelastic DM model.

\section{A. Lifetime of the heavier DM component $\boldsymbol{X}^{\prime}$}

To resolve the XENON1T anomaly by inelastic DM, it is important to ensure that the lifetime of the heavier DM component $X^{\prime}$ is longer than the age of the Universe. Since we have the DM mass splitting $\Delta m_{X} \ll m_{e}$, only the decays such as $X^{\prime} \rightarrow X \gamma \gamma$ and $X^{\prime} \rightarrow X \nu \bar{\nu}$ are kinematically allowed. The decay channel $X^{\prime} \rightarrow X \gamma \gamma$ arises from one-loop diagrams with electrons $e_{R}$ or quarks $u_{R}\left(d_{R}\right)$ running in the loop. Thus, with Refs. [8,21], we can estimate the partial decay width of $X^{\prime}$ as

$$
\Gamma_{X^{\prime} \rightarrow X \gamma \gamma} \simeq \sum_{f=e, u, d} \frac{\alpha^{2} g_{X}^{4} q_{\mathrm{DM}}^{2}}{155520 \pi^{5}}\left(\frac{q_{f} N_{c f}}{m_{f}^{2}}\right)^{2} \frac{\Delta m_{X}^{9}}{m_{A^{\prime}}^{4}},
$$

where the sum runs over the electron loop $(f=e)$ and quark loops $(f=u, d), q_{f}$ denotes the electric charge of each fermion $f$, and the color factor $N_{c f}=1(3)$ for the electron (quark) loop. Since the quark masses $m_{u, d} \approx$ (1.5-6) $\mathrm{MeV}$ are much larger than the electron mass with the mass ratio $\left(m_{e} / m_{u, d}\right)^{4}=O\left(10^{-2}-10^{-4}\right)$, we see that the electron loop dominates the partial decay width in Eq. (16). From fitting the XENON1T event rate, we have a small DM mass splitting $\Delta m_{X} \simeq 3 \mathrm{keV}$ and the ratio of $m_{A^{\prime}} / g_{X}$ given by Eq. (13). This leads to a tiny partial decay width $\Gamma_{X^{\prime} \rightarrow X \gamma \gamma} \approx\left(3 \times 10^{24} \mathrm{yr}\right)^{-1}$, and thus $\Gamma_{X^{\prime} \rightarrow X \gamma \gamma}^{-1} \ggg \tau_{U}$, where $\tau_{U} \simeq 1.38 \times 10^{10} \mathrm{yr}$ is the age of the present Universe. Such a long lifetime is also much beyond the x-ray [22] and CMB [23] constraints on the lifetime of radiatively decaying DM. These observations typically exclude DM with a lifetime shorter than $10^{20} \mathrm{yr}$.

\footnotetext{
${ }^{4}$ Note that for DM mass $\lesssim 1 \mathrm{GeV}$, the typical recoil energy of scattering off a nucleus is below $1 \mathrm{keV}$. We thus only include the down-scattering process $X^{\prime}+N \rightarrow X+N$ for the bound.
} 

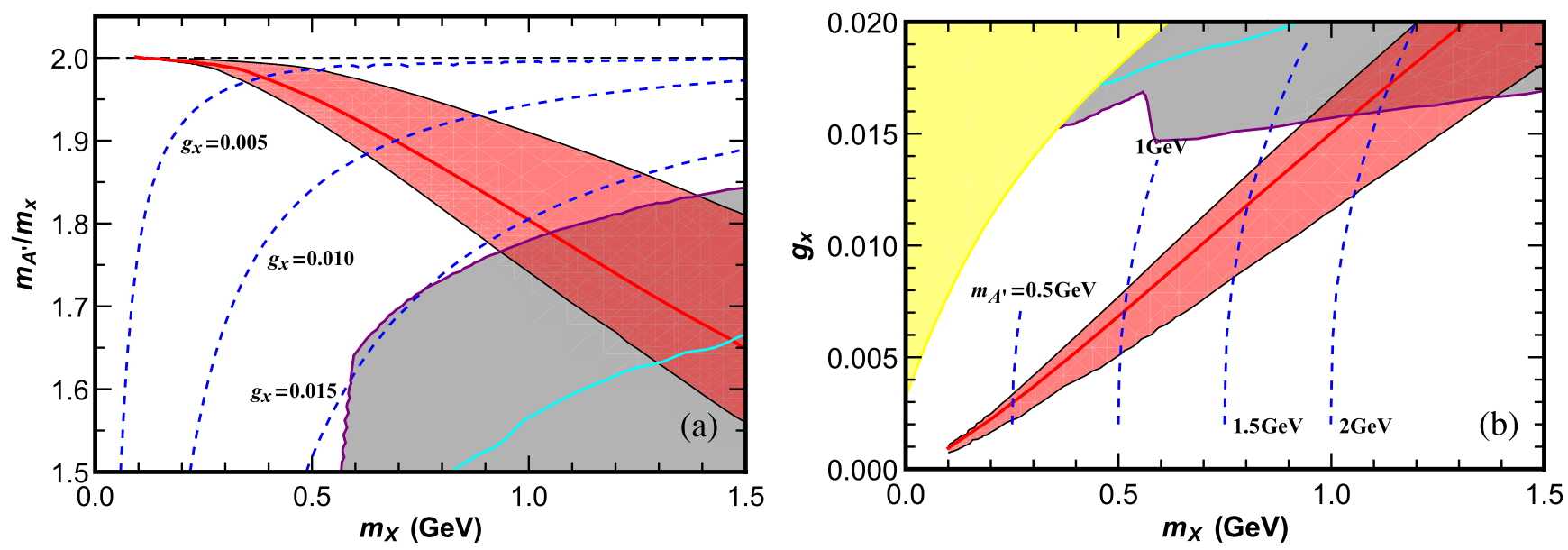

FIG. 2. Allowed parameter space for the inelastic scalar DM. In each plot, the pink areas depict the allowed regions (95\% C.L.) by the XENON1T electron recoil data [2] combined with the DM relic density bound, and the red solid curve gives the best-fit values. The gray areas are excluded by nucleon recoil detections of low threshold (90\% C.L.), where the purple and light blue curves are exclusion limits by XENON1T [11] and CRESST-III [12], respectively. (a) Constraints on the parameter space of $m_{X}$ versus $m_{A^{\prime}} / m_{X}$. The blue dashed curves show the allowed parameter space of realizing the observed DM relic density for a set of sample $g_{X}$ values. (b) Constraints on the parameter space of $m_{X}$ versus $g_{X}$. The blue dashed curves show the parameter space of realizing the observed DM relic density for a set of sample mediator masses $m_{A^{\prime}}$. The yellow region is excluded by the DM relic density bound for $m_{A^{\prime}} / m_{X}<2$.

The other decay channel, $X^{\prime} \rightarrow X \nu \bar{\nu}$, occurs through the $A^{\prime}$ exchange due to the neutral gauge boson mixing matrix [Eq. (5)]. The contribution of the $Z$ exchange as induced by the mixing matrix in Eq. (5) is fully negligible because of the large suppression factor $\left(m_{A^{\prime}}^{4} / M_{Z}^{4}\right) \lesssim O\left(10^{-4}\right)$ relative to the $A^{\prime}$ exchange. Thus, we can estimate the $X^{\prime}$ decay width as follows:

$$
\Gamma_{X^{\prime} \rightarrow X \nu \bar{\nu}} \simeq \frac{q_{\mathrm{DM}}^{2} g_{X}^{4}}{160 \pi^{3}} \frac{v_{1}^{4} \Delta m_{X}^{5}}{v_{h}^{4} m_{A^{\prime}}^{4}},
$$

where we have included the contributions of the final-state neutrinos from three families. The result holds for both scalar and fermionic DM. By requiring that the $X^{\prime}$ lifetime be much longer than the age of our present Universe, $\Gamma_{X^{\prime} \rightarrow X \nu \bar{\nu}}^{-1} \gtrsim 10 \tau_{U} \gg \tau_{U}$, we derive a constraint:

$$
v_{1} \lesssim 17 \mathrm{GeV} \times\left(\frac{1 \mathrm{GeV}}{m_{X}}\right)^{\frac{1}{4}} .
$$

This shows that in our model, the electroweak symmetry breaking (EWSB) is mainly generated by the Higgs doublet $H_{2}$, and the VEV ratio $\tan \beta=v_{2} / v_{1} \gtrsim 10$.

\section{B. Dark matter relic abundance}

Since our construction always holds $\Delta m_{X} \ll m_{X}$, the two DM components can be regarded as a degenerate complex scalar or Dirac spinor before they freeze out. We thus determine the relic density of a complex scalar $\hat{X}$ or a Dirac spinor $\hat{\chi}$. We compute the DM relic density of the current model by using the package MicrOMEGAs $[15,24]$ and present our results for the scalar DM model in Fig. 2. In Fig. 2(a), the blue curves show the masses of $X\left(X^{\prime}\right)$ and $A^{\prime}$ which achieve the observed DM relic abundance for each given value of the gauge coupling $g_{X}$. The pink area presents the allowed regions (95\% C.L.) by the XENON1T data [2] combined with the DM relic density bound, and the red solid curve depicts the best fit. The gray region is excluded by the XENON1T [11] and CRESST-III [12] measurements combined with the DM relic density bound. We find that the DM mass range $m_{X} \lesssim 1.4 \mathrm{GeV}$ is favored in our model, and in the viable parameter space, the dominant DM annihilation channel is $\hat{X}^{*} \hat{X} \rightarrow A^{\prime} \rightarrow f_{R} \bar{f}_{R}$, where $f=u, d, e$. In Fig. 2(b), the pink region presents the allowed parameter space of $m_{X}$ versus $g_{X}$ at a $95 \%$ C.L., and the red solid curve gives the best-fit values. The blue curves show the allowed values of the gauge coupling $g_{X}$, as a function of the DM mass $m_{X}$, which achieve the observed DM relic abundance for each given mediator mass $m_{A^{\prime}}$. We fit the XENON1T electron recoil data [2] and DM relic density bound together, and include the constraints of $m_{X} \lesssim 1.4 \mathrm{GeV}$ by XENON1T [11] and CRESST-III [12] nuclear recoil measurements (the gray area). With these, we derive the combined limit $g_{X} \lesssim 0.016$ for the case $m_{A^{\prime}} / m_{X}<2$.

In Fig. 3, we present our results for the inelastic fermionic DM. Similarly to the scalar DM case, the annihilation of fermionic DM in the viable parameter space is dominated by the $s$-channel process $\hat{\chi}^{\dagger} \hat{\chi} \rightarrow A^{\prime} \rightarrow f_{R} \bar{f}_{R}$. But the annihilation is more efficient in the case of fermionic DM because it is $s$-wave dominant; whereas for the scalar DM, the process is $p$-wave dominant due to its derivative coupling. In order to achieve the observed 

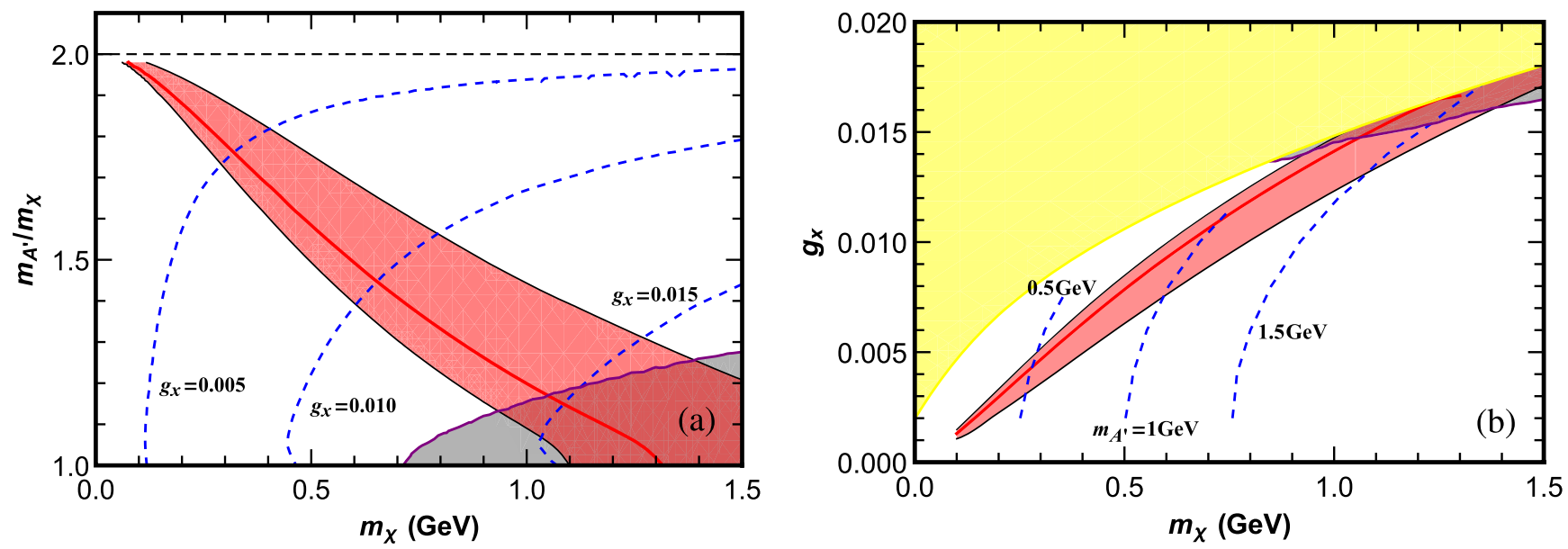

FIG. 3. Allowed viable parameter space for the inelastic fermionic DM, as shown in plot (a) for $m_{X}$ versus $m_{A^{\prime}} / m_{X}$, and in plot (b) for $m_{X}$ versus $g_{X}$. The curves and shaded regions have the same meanings as defined in the caption of Fig. 2, except that the current plots present the case of the inelastic fermionic DM.

DM relic density, the parameters for the fermionic DM have to be farther away from the resonance region than that for the scalar DM. Hence, in Fig. 3(a), the viable parameter region (pink area) for the fermionic DM covers lower values of the mass ratio $m_{A^{\prime}} / m_{\chi}$, as compared to that in Fig. 2(a).

\section{Current ratio of $X$ and $X^{\prime}$}

Next, we examine whether the constrained couplings in Figs. 2 and 3 are consistent with the condition $n_{X}=n_{X^{\prime}}$. After the decoupling of the annihilation process $\hat{X} \hat{X}^{\dagger} \rightarrow f_{R} \bar{f}_{R}$, the total DM number density $n_{\hat{X}}=n_{X}+n_{X^{\prime}}$ is fixed. However, $X$ and $X^{\prime}$ can still convert into each other via the processes $e^{ \pm} X \leftrightarrow e^{ \pm} X^{\prime}$ and $X^{\prime} X^{\prime} \leftrightarrow X X$. As we showed previously [8], the former process decouples at $T \sim m_{e}\left(\gg \Delta m_{X}\right)$ for the GeV-scale DM. The latter process is induced by $t$ - and $u$-channel exchanges of $A^{\prime}$. If the annihilation $X^{\prime} X^{\prime} \rightarrow X X$ decoupled at a temperature $T^{\prime}<\Delta m_{X}$, the $X^{\prime}$ density would be exponentially suppressed by a factor of $\exp \left(-\Delta m_{X} / T^{\prime}\right)$. As an estimate, this process becomes inefficient when the reaction rate $\Gamma\left(T^{\prime}\right) \lesssim H\left(T^{\prime}\right)$. We estimate this reaction rate as $\Gamma\left(T^{\prime}\right) \simeq$ $\left\langle\sigma^{\prime} v_{\mathrm{DM}}\right\rangle n_{\mathrm{DM}}$, in which the thermally averaged DM annihilation cross section $\left\langle\sigma^{\prime} v_{\mathrm{DM}}\right\rangle \simeq g_{X}^{4} q_{\mathrm{DM}}^{4} \sqrt{m_{X}^{3} T^{\prime}} /\left(\pi m_{A^{\prime}}^{4}\right)$ and the DM density $n_{\mathrm{DM}} \simeq T_{\mathrm{eq}} T^{\prime 3} / m_{X}$. Here, $T_{\text {eq }}$ is the temperature at matter-radiation equality. Thus, with these and Eq. (13), we can estimate

$$
T^{\prime} \approx 0.01 \mathrm{GeV} \times\left(\frac{1 \mathrm{GeV}}{m_{X}}\right) \times\left(\frac{3}{q_{\mathrm{DM}}}\right)^{\frac{4}{3}} .
$$

Shortly after the kinetic decoupling, the DM temperature drops rapidly as $a(t)^{-2}$ and falls below $\Delta m_{X}$ quickly. The $X^{\prime}$ density would get depleted if the annihilation $X^{\prime} X^{\prime} \rightarrow$ $X X$ were still efficient. As a conservative estimate, we demand $T^{\prime} \gtrsim m_{e}$, so the process $X^{\prime} X^{\prime} \rightarrow X X$ decouples before the kinetic decoupling between $e^{ \pm}$and the DM. This imposes an upper bound $m_{X} \lesssim 20(50) \mathrm{GeV}$ for scalar (fermionic) DM, which is always satisfied in our models.

\section{ANALYZING LABORATORY CONSTRAINTS}

In this section, we proceed to analyze the relevant laboratory constraints on our inelastic DM models.

\section{A. Correction to $Z$-boson mass}

Our model contributes to several electroweak precision observables. Using Eq. (4), we derive the new correction to Z-boson mass:

$$
\delta M_{Z}=\frac{g_{X}^{2} v_{1}^{4}}{4 v_{h}^{2} M_{Z}}
$$

The $Z$ pole observables have been measured with high precision. Especially, fitting the SM with the precision data gives the prediction $M_{Z}^{\mathrm{sm}}=(91.1884 \pm 0.0020) \mathrm{GeV}$, while the direct measurement of the $Z$-boson mass gives $M_{Z}^{\exp }=(91.1876 \pm 0.0021) \mathrm{GeV}$ [25]. This strongly constrains any new physics contribution to the $Z$ mass: $\delta M_{Z}<$ $0.0049 \mathrm{GeV}$ at a $95 \%$ C.L. Thus, we derive an upper bound on the VEV of the Higgs doublet $H_{1}$ :

$$
v_{1} \lesssim 15.2 \mathrm{GeV} / \sqrt{g_{X}} .
$$

\section{B. Correction to electron anomalous magnetic moment}

The $U(1)_{X}$ interaction also alters the anomalous magnetic moments of electrons. For $m_{A^{\prime}} \gg m_{e}$, we derive the one-loop correction to $a_{e}=\frac{1}{2}\left(g_{e}-2\right)$ : 


$$
\delta a_{e}^{X} \simeq-\frac{g_{X}^{2} q_{e}^{2}}{12 \pi^{2}} \frac{m_{e}^{2}}{m_{A^{\prime}}^{2}},
$$

with $q_{e}=-\frac{1}{2}$, which agrees with Ref. [26]. We note that $\delta a_{e}^{X}<0$ because the mediator $A^{\prime}$ couples to electrons via right-handed coupling only. In contrast, the kinetically mixed dark photon model generally predicts positive correction to $a_{e}$ due to its vectorlike coupling to the electron. For $\left(g_{X}, m_{A^{\prime}}\right)$ obeying Eq. (13) and for the scalar (fermionic) DM having $U(1)_{X}$ charge $q_{\mathrm{DM}}=3(3 / 2)$, we derive the correction to the electron anomalous magnetic moment:

$$
\delta a_{e}^{X} \simeq-3.8(7.6) \times 10^{-14} \times \sqrt{m_{X} /(1 \mathrm{GeV})} .
$$

These are around the same order of magnitude as the current experimental sensitivity of $2 \times 10^{-13}$ [25] and can be further probed by the future precision measurement of electron magnetic dipole moment, especially for the fermionic inelastic DM scenario.

\section{Other constraints on the Higgs sector}

There are additional bounds that can constrain the Higgs sector of our inelastic DM model. For instance, the signal strength of the SM-like Higgs boson $h(125 \mathrm{GeV})$ measured at the LHC can constrain the mixing between the $C P$ even neutral component of $\mathrm{H}_{2}$ and the other scalar singlets. The flavor-dependent feature of our two-Higgs-doublet sector could induce the flavor-changing processes via Higgs exchange, so the mass of the heavy neutral Higgs (mainly from the $H_{1}$ doublet) is constrained by meson mixings. Since the present study focuses on analyzing the DM and its vector portal to the SM particles, we note that these constraints can be satisfied by proper parameter choice in the Higgs sector, which will be elaborated in Appendix B.

\section{Collider constraints}

Since the mediator $A_{\mu}^{\prime}$ couples directly to the righthanded electrons, our model will receive nontrivial tests by the $e^{+} e^{-}$collider measurements. (There are discussions on constraining light DM models at lepton colliders in the literature [27-29].) The mediator $A_{\mu}^{\prime}$ contributes constructively to the cross section of $e^{+} e^{-} \rightarrow e^{+} e^{-}$. This cross section has been measured by LEP [30], with which we infer a bound on our model, $\sqrt{4 \pi s} /\left(\left|q_{e}\right| g_{X}\right)>8.6 \mathrm{TeV}$ at 95\% C.L., ${ }^{5}$ where $\sqrt{s} \simeq 200 \mathrm{GeV}$ is the LEP collider energy, and $q_{e}=-\frac{1}{2}$ is the $U(1)_{X}$ charge of $e_{R}$ in our model. From this, we deduce an upper bound on the $U(1)_{X}$ gauge coupling, $g_{X} \lesssim 0.16$.

\footnotetext{
${ }^{5}$ Hereafter, all the quoted experimental bounds are set at $95 \%$ C.L. unless specified otherwise.
}

The DM particles can be pair-produced in $e^{+} e^{-}$collisions through $s$-channel $A^{\prime}(Z)$ exchanges and in association with the final-state monophoton, $e^{+} e^{-} \rightarrow X X^{\prime} \gamma$. The rate of the DM production with a monophoton via $Z$ exchange is highly suppressed by a coupling factor $v_{1}^{4} / v_{h}^{4}$ which arises from the gauge boson mixing matrix [Eq. (5)]. As we have shown in Sec. IV and Figs. 2 and 3, the $A^{\prime}$ mass has to be less than a few $\mathrm{GeV}$ due to the combined bounds of realizing the DM relic density and fitting the DM directdetection data. We find that for the contribution of $A^{\prime}$ exchange in the case of $\sqrt{s}>m_{A^{\prime}}>2 m_{X}$ at LEP [31], the cross section of this process has a resonance at $E_{\gamma} / E_{\text {beam }} \sim 1-m_{A^{\prime}}^{2} / s$. By fitting the LEP monophoton data with parameters obeying the relic density bound, we derive the $95 \%$ exclusion limit on our models, $g_{X} \lesssim 0.015$. On the other hand, we find that in the parameter region $m_{A^{\prime}}<2 m_{X}$ and under the DM relic density bound, the contribution to the LEP monophoton process is too small to receive constraint, so this region is always allowed.

The monophoton searches at low-energy $e^{+} e^{-}$colliders such as BABAR [32] and Belle-II [33] can also place nontrivial bounds on models of light DM [28]. We summarize these bounds on our model as follows, according to Ref. [28]: For the mass region $m_{X} \lesssim 1 \mathrm{GeV}$ and $m_{A^{\prime}} \lesssim 2 m_{X}$, the $B A B A R$ measurements constrain $g_{X}^{2}\left|q_{e}\right| q_{\mathrm{DM}}<O\left(10^{-2}\right)$. For the ongoing Belle-II experiment, the projected constraint set by null result is $g_{X}^{2} q_{e} q_{\mathrm{DM}}<O\left(10^{-3}\right)$, assuming that the backgrounds are ideally known. As shown in Sec. IV, for this case, combining the constraints from the DM direct detections and realizing the DM relic density will require $g_{X} \lesssim 0.016$, which satisfies all the $e^{+} e^{-}$collider bounds. For the mass range $m_{A^{\prime}}>2 m_{X}$, the $B A B A R$ experiment sets a strong constraint on the $A^{\prime}-e^{ \pm}$coupling. It is stronger than the LEP monophoton search limit, due to the much larger production cross section and the much higher integrated luminosity of $B A B A R$. Given our charge assignment $q_{e}=1 / 2$ and the branching fraction of $A^{\prime} \rightarrow X X^{\prime}$, we can derive the corresponding constraint on our model, $g_{X} \lesssim 0.003$. Combined with the bounds from the XENON1Telectron recoil and DM relic density, the $B A B A R$ bound on $g_{X}$ further constrains the DM and mediator masses $m_{X}<0.25 \mathrm{GeV}$ and $m_{A^{\prime}}<0.68 \mathrm{GeV}$ for the inelastic scalar DM, and $m_{X}<0.21 \mathrm{GeV}$ and $m_{A^{\prime}}<0.55 \mathrm{GeV}$ for the inelastic fermionic DM. Thus, the $m_{A^{\prime}}>2 m_{X}$ region is largely excluded.

The LHC measurement of $Z \rightarrow 4 \mu$ decays [34] can constrain the coupling between $A_{\mu}^{\prime}$ and $\mu^{ \pm}$. This coupling is induced from the neutral gauge boson mixing matrix [Eq. (5)], and the corresponding Lagrangian term is

$$
\mathcal{L} \supset \frac{g_{X} v_{1}^{2}}{v_{h}^{2}} \bar{\mu} \gamma^{\mu}\left[\left(\sin ^{2} \theta_{W}-\frac{1}{4}\right)+\frac{1}{4} \gamma^{5}\right] A_{\mu}^{\prime} \mu .
$$


For a vector-type new interaction $g_{\text {new }} \bar{\mu} \gamma^{\mu} A_{\mu}^{\prime} \mu$ and the small mediator mass $m_{A^{\prime}}<10 \mathrm{GeV}$, the LHC has placed a bound on its coupling, $g_{\text {new }} \lesssim 4.5 \times 10^{-3}$ at $95 \%$ C.L. For $v_{1}^{2} / v_{h}^{2}=10^{-2}$, we convert this LHC bound to a constraint on the $U(1)_{X}$ coupling of our model, $g_{X} \lesssim O(1)$, which is weaker than the combined bound by the DM direct detections and the DM relic density. The inelastic DM particles of our model can also be directly produced at the LHC through its coupling to the right-handed quarks $\left(u_{R}, d_{R}\right)$, giving raise to monophoton signals together with the missing $P_{T}$. Such signals have been actively searched by ATLAS [35] and CMS [36], but these searches lose sensitivity for $g_{X}<O(0.1)$ [37] and do not constrain the cosmologically favored parameter space as discussed in Sec. IV.

\section{CONCLUSIONS}

In this work, we proposed a new candidate of $\mathrm{GeV}$-scale inelastic dark matter (DM), which can be either scalars or fermions. For this we constructed anomaly-free and renormalizable inelastic DM models under a new $U(1)_{X}$ gauge symmetry with a dark photon mediator (without assuming kinetic mixing) and with scalar or fermionic DM particles (cf. Table I). We realized the natural $O(\mathrm{keV})$ mass splitting for the inelastic DM by a scalar seesaw mechanism. Our model resolved the recent XENON1T anomaly in the electron recoil detection [2]. It is highly predictive and testable. We further analyzed the nontrivial bounds from the nuclear recoil detection (including the Migdal effect) by the XENON1T [11], CRESST-III [12], CDEX-1B [13], and DarkSide-50 [14] experiments with low recoil energy thresholds. Combining the constraints from both the electron recoil and nuclear recoil detections, we identified the viable parameter space and predicted the inelastic DM mass $\lesssim 1.4 \mathrm{GeV}$, as shown in Fig. 1(b). Then, we derived the viable parameter space in Fig. 2 for the scalar inelastic DM and Fig. 3 for the fermionic inelastic $\mathrm{DM}$, under the constraints by the DM relic abundance, the lifetime of the heavier DM component, the electroweak precision tests, and the collider searches. The upcoming DM direct-detection experiments by the PandaX-4T [38], LZ [39], and XENONnT [40] Collaborations will provide decisive probes of our $\mathrm{GeV}$-scale inelastic DM candidate.

\section{ACKNOWLEDGMENTS}

We thank Jianglai Liu, Qian Yue, and Ning Zhou for useful discussions on DM direct detections. This research was supported in part by the National NSF of China (under Grants No. 11835005 and No. 12175136), by the National Key R\&D Program of China (under Grant No. 2017YFA0402204), and by the CAS Center for Excellence in Particle Physics (CCEPP). It was also supported in part by the Office of Science and Technology, Shanghai Municipal Government.

\section{APPENDIX A: ESTIMATE OF IMPROVED CONSTRAINT OF THE MIGDAL EFFECT ON THE INELASTIC DARK MATTER}

In this appendix, we make an estimate of the improved constraint on the inelastic DM by using a scaling relation of the Migdal effect process.

We begin by recalling the formulation of the Migdal process for the direct detection of a single component DM [19]. The differential rate of electron recoil (ER) in the Migdal process per unit target mass is given by

$\frac{\mathrm{d} R}{\mathrm{~d} E_{\mathrm{ER}}} \simeq \int \mathrm{d} E_{\mathrm{NR}} \mathrm{d} v\left[\frac{\mathrm{d}^{2} R_{\mathrm{N}}}{\mathrm{d} E_{\mathrm{NR}} \mathrm{d} v} \sum_{n, \ell} \frac{1}{2 \pi} \frac{\mathrm{d} P_{q_{e}}^{c}\left(n, \ell \rightarrow E_{e}\right)}{\mathrm{d} E_{\mathrm{ER}}}\right]$,

where the differential rate of the nuclear recoil per unit target mass is

$$
\frac{\mathrm{d} R_{\mathrm{N}}}{\mathrm{d} E_{\mathrm{NR}} \mathrm{d} v} \simeq \frac{\rho_{X} \sigma_{A}}{2 m_{X} \mu_{A}^{2}} \frac{f(v)}{v} .
$$

In the above, $\rho_{X}$ is the local DM density, $\mu_{A}$ is the DM-atom reduced mass, and $\sigma_{A} \equiv \frac{\left|F_{A}\right|^{2}|\mathcal{M}|^{2}}{\left(m_{A}+m_{X}\right)^{2}}$ is a parametrization of the atomic form factor $F_{A}$ and the amplitude. It equals the DM-nuclear cross section for elastic scattering, but not for the inelastic case. The function $f(v)$ is the distribution of the local DM velocity $v$. The value $P_{q_{e}}^{c}\left(n, \ell \rightarrow E_{e}\right)$ is the probability of exciting an $(n, \ell)$ electron with ionization energy $E_{n, \ell}$ to an unbounded electron with energy deposit $E_{e}=E_{\mathrm{ER}}-E_{n, \ell}$. The momentum $q_{e}=m_{e} v_{A}$, where $v_{A}$ is the atom velocity after scattering in the lab frame. We can further write

$$
\frac{1}{2 \pi} \frac{\mathrm{d} P_{q_{e}}\left(n, \ell \rightarrow E_{e}\right)}{\mathrm{d} E_{e}} \equiv q_{e}^{2} A_{n, \ell}\left(E_{e}\right)
$$

where $q_{e}^{2}=2 m_{e}^{2} E_{\mathrm{NR}} / m_{A}$. Thus, $A_{n, \ell}\left(E_{e}\right)$ is only a function of $E_{e}$ [19], but it is independent of $E_{\mathrm{NR}}$.

In general, the detected energy $E_{\mathrm{det}}$ receives contributions from both nuclear and electron recoil, $E_{\mathrm{det}}=$ $E_{\mathrm{ER}}+\mathcal{L} E_{\mathrm{NR}}$, with $\mathcal{L} \approx 0.15$ as the quenching factor of nuclear recoil. For small $m_{X} \lesssim 1 \mathrm{GeV}$ and $\delta=O(\mathrm{keV})$, the nuclear recoil energy $E_{\mathrm{NR}} \lesssim \frac{\mu_{A}}{m_{A}}\left(\frac{1}{2} \mu_{A} v^{2}+\delta\right)$ is rather small, so its contribution to the detected energy can be neglected in the following estimate for the sensitive range of XENON1T with $E_{\mathrm{det}} \simeq E_{\mathrm{ER}} \gtrsim 0.1 \mathrm{keV}$.

For the spin-independent interaction, the DM-nuclear cross section $\sigma_{A}$ is independent of the nuclear recoil energy. 
We can perform the integration over $E_{\mathrm{NR}}$ analytically in Eq. (A1), and obtain the result ${ }^{6}$

$$
\begin{aligned}
\frac{\mathrm{d} R}{\mathrm{~d} E_{\mathrm{ER}}} & =\frac{1}{m_{A}} \frac{\rho_{X} \sigma_{A} m_{e}^{2} \mu_{A}^{2}}{m_{X} m_{A}^{2}} A_{n, \ell}\left(E_{\mathrm{ER}}-E_{n, \ell}\right) \\
& \times 2 \int_{v_{\min }} \mathrm{d} v f(v) v \sqrt{1+\frac{2\left(\delta-E_{\mathrm{ER}}\right)}{\mu_{A} v^{2}}}\left(\frac{\delta-E_{\mathrm{ER}}+\mu_{A} v^{2}}{\mu_{A}}\right) .
\end{aligned}
$$

For a fixed $E_{\mathrm{ER}}$, the minimum velocity $v_{\min }\left(E_{\mathrm{ER}}\right)$ to excite the electron is given by

$$
v_{\min }\left(E_{\mathrm{ER}}\right)^{2}=\max \left[\frac{2\left(E_{\mathrm{ER}}-\delta\right)}{\mu_{A}}, 0\right],
$$

where $v_{\min }\left(E_{\mathrm{ER}}\right)=0$ for $E_{\mathrm{ER}}<\delta$. For $\delta \simeq 2.8 \mathrm{keV}$ considered in the present study, the major part of the recoil spectrum of the Migdal process lies within $E_{\mathrm{ER}} \lesssim 1 \mathrm{keV}$ [20]. As an estimate, we set $v$ as the most probable velocity $v_{0} \approx 0.77 \times 10^{-3}$ of the local DM. Thus, we obtain an approximate differential spectrum for $\mu_{A} \approx m_{X}$ :

$$
\frac{\mathrm{d} R}{\mathrm{~d} E_{\mathrm{ER}}} \propto \frac{\mu_{A}^{2}}{\mu_{N}^{2}} \sigma_{N} \sqrt{1+\frac{2\left(\delta-E_{\mathrm{ER}}\right)}{\mu_{A} v_{0}^{2}}}\left(\delta-E_{\mathrm{ER}}+\mu_{A} v_{0}^{2}\right) .
$$

Here, $\mu_{N}$ is the DM-nucleon reduced mass and $\sigma_{N}$ the DMnucleon cross section. To utilize the direct-detection results, we have adopted the convention that DM is coupled equally to neutrons and protons in deriving Eq. (A6), and thus ${ }^{7}$ $\sigma_{A} / \sigma_{N}=A^{2} \mu_{A}^{2} / \mu_{N}^{2}$.

For the light DM, the difference $\delta-E_{\mathrm{ER}} \gg \mu_{A} v^{2}$ and the recoil spectrum scales as $\sqrt{1 / m_{X}}$.

So far in the literature, the constraints from including the Migdal effect are only given for elastic DM and for inelastic DM with a few special inputs of $\delta$ [20]. We estimate the constraint on the (exothermic) inelastic DM candidate with $\delta=2.8 \mathrm{keV}$ (cf. main text) based on the following observations. Reference [20] shows that the detectable sides of the spectrums are similar for elastic and inelastic DM with

\footnotetext{
${ }^{6}$ We have evaluated Eq. (A4) numerically and confirmed its good agreement with most curves in Figs. 2 and 3 of Ref. [20] for $E_{\mathrm{ER}} \gtrsim 0.1 \mathrm{keV}$. We obtained a less suppressed exothermic scattering spectrum in the range $E_{\mathrm{ER}} \gtrsim 1 \mathrm{keV}$ for a DM of $7 \mathrm{MeV}$ mass in comparison with the green curve in Fig. 3 of Ref. [20]. We have confirmed this with Jayden Newstead (the coauthor of Ref. [20]), and this discrepancy can be traced back to a minor coding error in producing the curves of Ref. [20]. We thank Jayden Newstead for clarification and for sharing his code of Ref. [20].

${ }^{7}$ The inelastic DM candidate studied in the main text couples to neutrons and protons with opposite charges. This requires additional treatment, as we have shown in Sec. III of our main text.
}

the parameters $\left[\delta / \mathrm{keV}, m_{X} / \mathrm{GeV}, \sigma_{N} /\left(10^{-40} \mathrm{~cm}^{2}\right)\right]=$ $(0,2,1)$ and $(4,0.5,0.65)$, respectively. According to the scaling relation of Eq. (A6), for $\delta=2.8 \mathrm{keV}$, the input parameters $\quad\left[m_{X} / \mathrm{GeV}, \quad \sigma_{N} /\left(10^{-40} \mathrm{~cm}^{2}\right)\right]=(2,0.41)$, $(1,0.83),(0.5,1.20)$, and $(0.1,1.13)$ give rise to the electron recoil spectra which are similar to those of $[\delta / \mathrm{keV}$, $\left.m_{X} / \mathrm{GeV}, \sigma_{N} /\left(10^{-40} \mathrm{~cm}^{2}\right)\right]=(4,0.5,0.65)$, and thus are also similar to those of elastic DM with $\left[m_{X} / \mathrm{GeV}\right.$, $\left.\sigma_{N} /\left(10^{-40} \mathrm{~cm}^{2}\right)\right]=(2,1)$. Since the constraint of the XENON1T Migdal effect is already given for the elastic DM cross section [11], we can estimate the constraint on the inelastic DM by rescaling the elastic DM constraint according to the parameters that give rise to similar spectra. We show our estimate by the purple dashed curve in Fig. 1(b).

\section{APPENDIX B: CONSTRAINTS ON THE HIGGS SECTOR}

In this appendix, we present the constraints on the Higgs sector of our model. For convenience, we denote the $C P$ even neutral components of $\left(H_{1}, H_{2}, S^{\prime}\right)$ as $\left(h_{1}, h_{2}, h_{S^{\prime}}\right)$, respectively. We consider the case of $M_{h_{1}}^{2} \gg M_{h_{2}}^{2}$ and $v_{1}^{2} \ll v_{2}^{2}$, so the observed Higgs boson $h(125 \mathrm{GeV})$ mainly contains the $h_{2}$ state. To realize these conditions, we consider the relevant part of the scalar potential,

$$
\begin{aligned}
V \supset & M_{H_{1}}^{2}\left|H_{1}\right|^{2}-M_{H_{2}}^{2}\left|H_{2}\right|^{2}-M_{S^{\prime}}^{2}\left|S^{\prime}\right|^{2}-M^{\prime} H_{1}^{\dagger} H_{2} S^{\prime} \\
& +\lambda_{1}\left|H_{1}\right|^{4}+\lambda_{2}\left|H_{2}\right|^{4}+\lambda_{3}\left|H_{1}\right|^{2}\left|H_{2}\right|^{2}+\lambda_{S^{\prime}}\left|S^{\prime}\right|^{4} \\
& +\lambda_{H_{1} S^{\prime}}\left|H_{1}\right|^{2}\left|S^{\prime}\right|^{2}+\lambda_{H_{2} S^{\prime}}\left|H_{2}\right|^{2}\left|S^{\prime}\right|^{2},
\end{aligned}
$$

where we only list terms relevant to $H_{1}, H_{2}$ and $S^{\prime}$. The cubic term $H_{1}^{\dagger} H_{2} S^{\prime}$ will ensure nonzero mass of the pseudoscalars. In Eq. (B1), we take all the mass parameters and the quartic couplings to be positive. We choose the Higgs doublet $H_{1}$ to have a positive mass term, so its mass $M_{H_{1}}$ can be naturally large and $M_{H_{1}}^{2} \gg v_{2}^{2}, v_{S^{\prime}}^{2}$. Thus, we deduce the VEV of $H_{1}$,

$$
v_{1} \simeq \frac{v_{2} v_{S^{\prime}} M^{\prime}}{M_{H_{1}}^{2}} .
$$

We see that requiring $M_{H_{1}}^{2} \gg M^{\prime} v_{S^{\prime}}$ can realize $v_{1}^{2} \ll v_{2}^{2}$. The LHC ATLAS and CMS experiments have measured the signal strength of the Higgs boson $h(125 \mathrm{GeV})$ for various channels, defined as $\mu_{h} \equiv\left\langle\sigma_{h} \cdot \mathrm{BR}\right\rangle_{\mathrm{obs}} /\left\langle\sigma_{h} \cdot \mathrm{BR}\right\rangle_{\mathrm{SM}}$, with $\sigma_{h}$ as the $h$-production cross section and BR as the decay branching fraction of a given channel. The most precisely measured decay channels are $\gamma \gamma$ and $W W^{*}$, which are consistent with the SM prediction $\left(\mu_{h}=1\right)$ to the $10 \%$ level [25]. This constrains the mixings of the SM-like Higgs boson $h_{2}$ with $h_{S^{\prime}}$ and $h_{1}$ down to $10 \%$ level. Note that for $v_{1}^{2} \ll v_{2}^{2}$, the $h_{2}-h_{1}$ mixing is mainly generated by 
the cubic term $-M^{\prime} H_{1}^{\dagger} H_{2} S^{\prime}$, and the contributions from the mixed quartic terms are suppressed by $v_{1} / M^{\prime}$. Thus, for $M_{H_{1}}^{2} \gg M_{H_{2}}^{2}$, the $h_{2}-h_{1}$ mixing is mainly determined by $\left|M^{\prime} v_{S^{\prime}} / M_{H_{1}}^{2}\right| \simeq v_{1} / v_{2}$. With this condition, we may choose the VEV ratio

$$
v_{1} / v_{2} \lesssim 0.1
$$

The small $h_{2}-h_{S^{\prime}}$ mixing can be induced by the contributions from the cubic and quartic terms with opposite signs. As an estimate, we ignore the small mixing of the heavy $h_{1}$ with the lighter states $h_{2}$ or $h_{S^{\prime}}$ and obtain

$$
\left|\sin \theta_{h_{2} h_{S^{\prime}}}\right| \simeq\left|\frac{\lambda_{H_{2} S^{\prime}} v_{2} v_{S^{\prime}}-M^{\prime} v_{1}}{M_{h_{S^{\prime}}}^{2}-M_{h}^{2}}\right| \ll 1,
$$

where $M_{h} \simeq 125 \mathrm{GeV}$ and $M_{h_{S^{\prime}}}$ are the mass eigenvalues of the $C P$-even neutral Higgs states $h$ and $h_{S^{\prime}}$.

Another constraint on the heavy Higgs mass $M_{H_{1}}$ comes from the flavor-changing processes mediated by the heavy scalars. The general Yukawa interactions for the quark sector take the following form:

$$
\begin{aligned}
\mathcal{L}= & -\sum_{i=1}^{3}\left(y_{u}^{i 1} \bar{Q}_{L i} \tilde{H}_{1} u_{R 1}+y_{d}^{i 1} \bar{Q}_{L i} H_{1} d_{R 1}\right) \\
& -\sum_{i=1,2,3}^{j=2,3}\left(y_{u}^{i j} \bar{Q}_{L i} \tilde{H}_{2} u_{R j}+y_{d}^{i j} \bar{Q}_{L i} H_{2} d_{R j}\right) \\
\equiv & -\bar{Q}_{L}^{i}\left(\overrightarrow{\mathbf{y}}_{\mathbf{u}} \tilde{H}_{1}, \hat{\mathbf{y}}_{\mathbf{u}} \tilde{H}_{2}\right)_{i j} u_{R j} \\
& -\bar{Q}_{L}^{i}\left(\overrightarrow{\mathbf{y}}_{\mathbf{d}} H_{1}, \hat{\mathbf{y}}_{\mathbf{d}} H_{2}\right)_{i j} d_{R j},
\end{aligned}
$$

where we denote $\tilde{H}_{i}=\mathrm{i} \sigma_{2} H_{i}^{*}$. For convenience, in the last line, we have decomposed the $3 \times 3$ Yukawa matrix $\mathbf{y}^{\mathbf{u}, \mathbf{d}}=\left(\overrightarrow{\mathbf{y}}_{\mathbf{u}, \mathbf{d}}, \hat{\mathbf{y}}_{\mathbf{u}, \mathbf{d}}\right)_{i j}$. Here, $\overrightarrow{\mathbf{y}}_{\mathbf{u}, \mathbf{d}}$ are $3 \times 1$ matrices of Yukawa couplings to $H_{1}$ and $\hat{\mathbf{y}}_{\mathbf{u}, \mathbf{d}}$ are $3 \times 2$ matrices of Yukawa couplings to $H_{2}$. The quarks acquire masses via Yukawa interactions with $H_{1}$ and $H_{2}$ taking their VEVs. The mass eigenstates are obtained by chiral rotations,

$$
\begin{array}{ll}
\mathbf{u}_{\mathbf{L}}^{\prime}=U_{L}^{u} \mathbf{u}_{\mathbf{L}}, & \mathbf{d}_{\mathbf{L}}^{\prime}=U_{L}^{d} \mathbf{d}_{\mathbf{L}}, \\
\mathbf{u}_{\mathbf{R}}^{\prime}=U_{R}^{u \dagger} \mathbf{u}_{\mathbf{R}}, & \mathbf{d}_{\mathbf{R}}^{\prime}=U_{R}^{d \dagger} \mathbf{d}_{\mathbf{R}},
\end{array}
$$

where $\mathbf{u}$ and $\mathbf{d}$ are vectors in flavor space denoting the third family of quarks, and $U_{L, R}^{u, d}$ are unitary rotation matrices. The quark mass matrices are diagonalized as

$$
\begin{aligned}
& U_{L}^{u \dagger}\left(\overrightarrow{\mathbf{y}}_{\mathbf{u}} v_{1}, \hat{\mathbf{y}}_{\mathbf{u}} v_{2}\right) U_{R}^{u}=v_{2} \mathbf{y}_{\mathbf{u}}^{\text {diag }}, \\
& U_{L}^{d \dagger}\left(\overrightarrow{\mathbf{y}}_{\mathbf{d}} v_{1}, \hat{\mathbf{y}}_{\mathbf{d}} v_{2}\right) U_{R}^{d}=v_{2} \mathbf{y}_{\mathbf{d}}^{\text {diag }},
\end{aligned}
$$

where $v_{2} \mathbf{y}_{\mathbf{u}(\mathbf{d})}^{\text {diag }}$ is the $3 \times 3$ diagonal mass matrix for up-type (down-type) quarks, whose diagonal elements give the measured quark masses. In the mass eigenbasis, the Yukawa interactions for the up-type quarks become

$$
\begin{aligned}
\mathcal{L}= & -\overline{\mathbf{u}}_{\mathbf{L}}^{\prime} U_{L}^{u^{\dagger}}\left(\overrightarrow{\mathbf{y}}_{\mathbf{u}} \tilde{H}_{1}^{0}, \hat{\mathbf{y}}_{\mathbf{u}} \tilde{H}_{2}^{0}\right) U_{R}^{u} \mathbf{u}_{\mathbf{R}}^{\prime} \\
= & -\overline{\mathbf{u}}_{\mathbf{L}}^{\prime} U_{L}^{u \dagger}\left(\overrightarrow{\mathbf{y}}_{\mathbf{u}}\left(\tilde{H}_{1}^{0}-\frac{v_{1}}{v_{2}} \tilde{H}_{2}^{0}\right),(\mathbf{0})\right) U_{R}^{u} \mathbf{u}_{\mathbf{R}}^{\prime} \\
& -\overline{\mathbf{u}}_{\mathbf{L}}^{\prime} \mathbf{y}_{\mathbf{u}}^{\operatorname{diag}} \tilde{H}_{2}^{0} \mathbf{u}_{\mathbf{R}}^{\prime},
\end{aligned}
$$

where $\tilde{H}_{1}^{0}$ and $\tilde{H}_{2}^{0}$ denote the neutral components of the Higgs doublets $\tilde{H}_{1}$ and $\tilde{H}_{2}$, respectively. Similarly, we can deduce the Yukawa interactions for the mass eigenstates of down-type quarks under the replacement $\mathbf{u} \rightarrow \mathbf{d}$ and $\tilde{H}_{i} \rightarrow H_{i}$. In Eq. (B8b), (0) denotes a $3 \times 2$ matrix in which all elements vanish. The first term in Eq. (B8b) would induce flavor-changing processes if the flavor-mixing matrices $U_{L, R}^{u}$ take an arbitrary pattern, and in this case it will receive strong constraints by the meson mixings. It is known that for well-motivated scenarios of flavor mixing, such constraints can be much reduced. For instance, we may consider a Cheng-Sher-like ansatz [41,42] on the flavor-changing Yukawa couplings,

$$
\xi_{u, d}^{i j}=\bar{\xi}_{u, d}^{i j} \times\left(\sqrt{m_{u, d}^{i} m_{u, d}^{j}} / v_{h}\right),
$$

where $(i, j)$ are family indices and the coupling coefficients $\bar{\xi}_{u, d}^{i j}$ can be naturally around $\bar{\xi}_{u, d}^{i j}=O(0.1-1)$. Thus, we set

$$
U_{L}^{u, d \dagger}\left(\overrightarrow{\mathbf{y}}_{\mathbf{u}, \mathbf{d}},(\mathbf{0})\right) U_{R}^{u, d}=\xi_{u, d}
$$

Then, the flavor-changing process between the $i$ th and $j$ th families is characterized by the new physics (NP) scale,

$$
\Lambda_{\mathrm{NP}}=\min \left(M_{h_{1}}, \frac{v_{2}}{v_{1}} M_{h_{2}}\right) \frac{\bar{\xi}_{u, d}^{i j} v_{h}}{\sqrt{m_{u, d}^{i} m_{u, d}^{j}}},
$$

where $M_{h_{1}}=O(5-10) \mathrm{TeV}$ is the mass of the heavy neutral Higgs state $h_{1}$ and $M_{h_{2}} \simeq 125 \mathrm{GeV}$ is the SM-like light Higgs boson $h_{2}$. For instance, the measurements of $K-\bar{K}$ mixing constrain $\Lambda_{\mathrm{NP}} \gtrsim 5 \times 10^{5} \mathrm{TeV}$ [43]. With this bound and for a natural coupling coefficient $\bar{\xi}_{d}^{12}=O(0.1)$, we obtain the limits on our Higgs sector, $M_{h_{1}} \gtrsim 6 \mathrm{TeV}$ and $v_{1} \lesssim 3.6 \mathrm{GeV}$. The $U(1)_{X}$ gauge boson $A_{\mu}^{\prime}$ also mediates the flavor-violating process, since it only couples to the right-handed quarks and leptons in the first family. The ratio $m_{A^{\prime}} / g_{X}$ is constrained by fitting the XENON1T data as in Eq. (13). So, the constraints from the meson-mixing measurements could be avoided by 
requiring the right-handed mass eigenstates $u_{R}^{\prime}$ and $d_{R}^{\prime}$ to be mainly aligned with the gauge eigenstates $u_{R}$ and $d_{R}$. In addition, we note that the flavor-violating effects can also occur in the lepton sector and induce flavor-violating leptonic decay channels for the SM-like light Higgs boson $h_{2}$. For instance, this leads to the interesting decay channel $h_{2} \rightarrow \mu^{ \pm} e^{\mp}$, which can be searched by analyzing the current LHC Run-2 data [44]. The upcoming LHC Run-3 and HL-LHC runs will have strong potential to discover this channel.
[1] E. Aprile et al. (XENON Collaboration), Eur. Phys. J. C 80, 785 (2020).

[2] E. Aprile et al. (XENON Collaboration), Phys. Rev. D 102, 072004 (2020).

[3] X. Zhou et al. (PandaX-II Collaboration), Chin. Phys. Lett. 38, 011301 (2021).

[4] A. E. Robinson, arXiv:2006.13278.

[5] K. Kannike, M. Raidal, H. Veermae, A. Strumia, and D. Teresi, Phys. Rev. D 102, 095002 (2020).

[6] L. Di Luzio, M. Fedele, M. Giannotti, F. Mescia, and E. Nardi, Phys. Rev. Lett. 125, 131804 (2020); F. Takahashi, M. Yamada, and W. Yin, arXiv:2006.10035; G. Alonso-Alvarez, F. Ertas, J. Jaeckel, F. Kahlhoefer, and L. J. Thormaehlen, arXiv:2006.11243; C. Boehm, D. G. Cerdeno, M. Fairbairn, P. A. N. Machado, and A. C. Vincent, arXiv:2006.11250; D. A. Sierra, V. De Romeri, L. J. Flores, and D. K. Papoulias, arXiv:2006.12457; B. Fornal, P. Sandick, J. Shu, M. Su, and Y. Zhao, arXiv:2006.11264; L. Su, W. Wang, L. Wu, J. M. Yang, and B. Zhu, arXiv:2006.11837; Y. Chen, J. Shu, X. Xue, G. Yuan, and Q. Yuan, arXiv:2006.12447; Q. H. Cao, R. Ding, and Q. F. Xiang, arXiv:2006.12767; H. Alhazmi, D. Kim, K. Kong, G. Mohlabeng, J. C. Park, and S. Shin, arXiv:2006.16252; Y. Jho, J. C. Park, S. C. Park, and P. Y. Tseng, arXiv:2006.13910; S. Chigusa, M. Endo, and K. Kohri, arXiv:2007.01663; J. Smirnov and J.F. Beacom, arXiv:2002.04038; A. Bally, S. Jana, and A. Trautner, arXiv:2006.11919; M. Du, J. Liang, Z. Liu, V. Q. Tran, and Y. Xue, arXiv:2006.11949; D. Aristizabal Sierra, V. De Romeri, L. J. Flores, and D. K. Papoulias, arXiv:2006.12457; N. F. Bell, J. B. Dent, B. Dutta, S. Ghosh, J. Kumar, and J. L. Newstead, arXiv:2006.12461; G. Paz, A. A. Petrov, M. Tammaro, and J. Zupan, arXiv:2006.12462; J. Buch, M. A. Buen-Abad, J. Fan, and J. S. C. Leung, arXiv:2006.12488; K. U. Dey, T. N. Maity, and T. S. Ray, arXiv:2006.12529; A. N. Khan, arXiv:2006.12887; K. Nakayama and Y. Tang, arXiv:2006.13159; L. Zu, G. W. Yuan, L. Feng, and Y. Z. Fan, arXiv:2006.14577; M. Lindner, Y. Mambrini, T. B. Melo, and F.S. Queiroz, arXiv:2006.14590; W. DeRocco, P. W. Graham, and S. Rajendran, arXiv:2006.15112; M. Chala and A. Titov, arXiv:2006.14596; C. Gao, J. Liu, L. T. Wang, X. P. Wang, W. Xue, and Y. M. Zhong, arXiv:2006.14598; J. B. Dent, B. Dutta, J. L. Newstead, and A. Thompson, arXiv:2006.15118; P. Ko and Y. Tang, Phys. Lett. B 815, 136181 (2021); W. Chao, Y. Gao, and M. Jin, arXiv:2006.16145; L. Delle Rose, G. Hütsi, C. Marzo, and L. Marzola, J. Cosmol. Astropart. Phys. 02 (2021) 031; B. Bhattacherjee and R. Sengupta, Phys. Lett. B 817, 136305 (2021); Y. Gao and T. Li,
arXiv:2006.16192; J. Sun and X. G. He, Phys. Lett. B 811, 135881 (2020); M. Szydagis, C. Levy, G. M. Blockinger, A. Kamaha, N. Parveen, and G. R. C. Rischbieter, Phys. Rev. D 103, 012002 (2021); T. Li, arXiv:2007.00874; O. G. Miranda, D. K. Papoulias, M. Tortola, and J. W. F. Valle, Phys. Lett. B 808, 135685 (2020); K. Benakli, C. Branchina, and G. Lafforgue-Marmet, Eur. Phys. J. C 80, 1118 (2020); N. Okada, S. Okada, D. Raut, and Q. Shafi, Phys. Lett. B 810, 135785 (2020); J. Davighi, M. McCullough, and J. Tooby-Smith, J. High Energy Phys. 11 (2020) 120; P. Athron et al., J. High Energy Phys. 05 (2021) 159; G. Arcadi, A. Bally, F. Goertz, K. Tame-Narvaez, V. Tenorth, and S. Vogl, Phys. Rev. D 103, 023024 (2021); C. Han, M. L. LopezIbanez, A. Melis, O. Vives, and J. M. Yang, Phys. Rev. D 103, 035028 (2021); Y. Ema, F. Sala, and R. Sato, Eur. Phys. J. C 81, 129 (2021); J. Kim, T. Nomura, and H. Okada, Phys. Lett. B 811, 135862 (2020); J. Cao, X. Du, Z. Li, F. Wang, and Y. Zhang, arXiv:2007.09981; D. Borah, S. Mahapatra, D. Nanda, and N. Sahu, Phys. Lett. B 811, 135933 (2020); S. Karmakar and S. Pandey, arXiv:2007.11892; S. Khan, Eur. Phys. J. C 81, 598 (2021); S. Shakeri, F. Hajkarim, and S. S. Xue, J. High Energy Phys. 12 (2020) 194; R. G. Cai, S. Sun, B. Zhang, and Y.L. Zhang, arXiv:2009.02315; R. Foot, arXiv:2011.02590; Y. Farzan and M. Rajaee, arXiv: 2007.14421.

[7] K. Harigaya, Y. Nakai, and M. Suzuki, Phys. Lett. B 809, 135729 (2020); H. M. Lee, arXiv:2006.13183; M. Baryakhtar, A. Berlin, H. Liu, and N. Weiner, arXiv:2006.13918; J. Bramante and N. Song, arXiv:2006.14089; I. M. Bloch, A. Caputo, R. Essig, D. Redigolo, M. Sholapurkar, and T. Volansky, arXiv:2006.14521; H. An and D. Yang, arXiv: 2006. 15672; S. Baek, J. Kim, and P. Ko, arXiv:2006.16876; A. Aboubrahim, M. Klasen, and P. Nath, arXiv:2011.08053; D. Borah, S. Mahapatra, and N. Sahu, arXiv:2009.06294.

[8] H. J. He, Y. C. Wang, and J. Zheng, J. Cosmol. Astropart. Phys. 01 (2021) 042.

[9] P. H. Gu and H. J. He, Phys. Rev. D 99, 015025 (2019); N. Okada and O. Seto, Phys. Rev. D 101, 023522 (2020).

[10] T. Gherghetta, J. Kersten, K. Olive, and M. Pospelov, Phys. Rev. D 100, 095001 (2019).

[11] E. Aprile et al. (XENON Collaboration), Phys. Rev. Lett. 123, 241803 (2019).

[12] A. H. Abdelhameed et al. (CRESST Collaboration), Phys. Rev. D 100, 102002 (2019).

[13] Z. Z. Liu et al. (CDEX Collaboration), Phys. Rev. Lett. 123, 161301 (2019).

[14] P. Agnes et al. (DarkSide Collaboration), Phys. Rev. Lett. 121, 081307 (2018). 
[15] G. Belanger, F. Boudjema, A. Pukhov, and A. Semenov, Comput. Phys. Commun. 180, 747 (2009).

[16] Z. H. Yu, J. M. Zheng, X. J. Bi, Z. Li, D. X. Yao, and H. H. Zhang, Nucl. Phys. B860, 115 (2012).

[17] E. Aprile et al. (XENON Collaboration), Phys. Rev. Lett. 121, 111302 (2018).

[18] P. Agnes et al. (DarkSide Collaboration), Phys. Rev. D 93, 081101 (2016).

[19] A. B. Migdal, J. Phys. (USSR) 4, 449 (1941); M. Ibe, W. Nakano, Y. Shoji, and K. Suzuki, J. High Energy Phys. 03 (2018) 194; M. J. Dolan, F. Kahlhoefer, and C. McCabe, Phys. Rev. Lett. 121, 101801 (2018).

[20] N. F. Bell, J. B. Dent, B. Dutta, S. Ghosh, J. Kumar, and J. L. Newstead, Phys. Rev. D 104, 7 (2021).

[21] C. B. Jackson, G. Servant, G. Shaughnessy, T. Tait, and M. Taoso, J. Cosmol. Astropart. Phys. 07 (2013) 021.

[22] R. Essig, E. Kuflik, S. D. McDermott, T. Volansky, and K. M. Zurek, J. High Energy Phys. 11 (2013) 193; O. Ruchayskiy, A. Boyarsky, D. Iakubovskyi, E. Bulbul, D. Eckert, J. Franse, D. Malyshev, M. Markevitch, and A. Neronov, Mon. Not. R. Astron. Soc. 460, 1390 (2016).

[23] T. R. Slatyer and C. L. Wu, Phys. Rev. D 95, 023010 (2017); L. Zhang, X. Chen, M. Kamionkowski, Z. G. Si, and Z. Zheng, Phys. Rev. D 76, 061301 (2007).

[24] G. Belanger, A. Mjallal, and A. Pukhov, Eur. Phys. J. C 81, 239 (2021).

[25] M. Tanabashi et al. (Particle Data Group), Phys. Rev. D 98, 030001 (2018).

[26] J. P. Leveille, Nucl. Phys. B137, 63 (1978).

[27] P. J. Fox, R. Harnik, J. Kopp, and Y. Tsai, Phys. Rev. D 84, 014028 (2011).

[28] R. Essig, J. Mardon, M. Papucci, T. Volansky, and Y. M. Zhong, J. High Energy Phys. 11 (2013) 167.
[29] L. Darmé, S. A. R. Ellis, and T. You, J. High Energy Phys. 07 (2020) 053.

[30] S. Schael et al. (ALEPH, DELPHI, L3, OPAL and LEP Electroweak Collaborations), Phys. Rep. 532, 119 (2013).

[31] J. Abdallah et al. (DELPHI Collaboration), Eur. Phys. J. C 38, 395 (2005); 60, 17 (2009).

[32] B. Aubert et al. (BABAR Collaboration), in Proceedings of the 34th International Conference in High Energy Physics (ICHEP-2008), Philadelphia, 2008, eConf C080730..

[33] T. Abe et al. (Belle-II Collaboration), arXiv:1011.0352.

[34] M. Aaboud et al. (ATLAS Collaboration), J. High Energy Phys. 06 (2018) 166; A. M. Sirunyan et al. (CMS Collaboration), Phys. Lett. B 792, 345 (2019).

[35] ATLAS Collaboration, Report No. ATLAS-CONF-2020048, CERN.

[36] CMS Collaboration, Phys. Lett. B 792, 345 (2019).

[37] O. Buchmueller, M. J. Dolan, S. A. Malik, and C. McCabe, J. High Energy Phys. 01 (2015) 037.

[38] H. Zhang et al. (PandaX Collaboration), Sci. China 62, 31011 (2019).

[39] D. Akerib et al. (LUX-ZEPLIN (LZ) Collaboration), Nucl. Instrum. Methods Phys. Res., Sect. A 953, 163047 (2020).

[40] E. Aprile et al. (XENON Collaboration), J. Cosmol. Astropart. Phys. 11 (2020) 031; 04 (2016) 027.

[41] For a review of the 2HDM, see G. C. Branco, P. M. Ferreira, L. Lavoura, M. N. Rebelo, M. Sher, and J. P. Silva, Phys. Rep. 516, 1 (2012).

[42] T. P. Cheng and M. Sher, Phys. Rev. D 35, 3484 (1987).

[43] C. Alpigiani, A. Bevan, M. Bona, M. Ciuchini, D. Derkach, E. Franco, V. Lubicz, G. Martinelli, F. Parodi, M. Pierini et al., arXiv:1710.09644.

[44] G. Aad et al. (ATLAS Collaboration), Phys. Lett. B 801, 135148 (2020). 